\title{
RELATIONS BETWEEN HIDDEN REGULAR VARIATION AND THE TAIL ORDER OF COPULAS
}

\author{
LEI HUA, ${ }^{*}$ Northern Illinois University \\ HARRY JOE,** University of British Columbia \\ HAIJUN LI, ${ }^{* * *}$ Washington State University
}

\begin{abstract}
We study the relations between the tail order of copulas and hidden regular variation (HRV) on subcones generated by order statistics. Multivariate regular variation (MRV) and HRV deal with extremal dependence of random vectors with Pareto-like univariate margins. Alternatively, if one uses a copula to model the dependence structure of a random vector then the upper exponent and tail order functions can be used to capture the extremal dependence structure. After defining upper exponent functions on a series of subcones, we establish the relation between the tail order of a copula and the tail indexes for MRV and HRV. We show that upper exponent functions of a copula and intensity measures of MRV/HRV can be represented by each other, and the upper exponent function on subcones can be expressed by a Pickands-type integral representation. Finally, a mixture model is given with the mixing random vector leading to the finite-directional measure in a product-measure representation of HRV intensity measures.
\end{abstract}

Keywords: Multivariate regular variation; tail dependence; upper exponent function; tail order function; intermediate tail dependence

2010 Mathematics Subject Classification: Primary 62H20

\section{Introduction}

Extremal dependence of a random vector can be described by the relative decay rate of certain joint tail probabilities of the random vector with respect to that of its margins. Such extremal dependence can be analyzed using multivariate regular variation (MRV) or hidden regular variation (HRV) (see Resnick (2007)), or, alternatively, using tail dependence or tail order functions of copulas. To the best of our knowledge, Jaworski (2004) first explicitly proposed the tail dependence functions for the bivariate case, and Jaworski (2006) studied an extension to the multivariate case. Li and Sun (2009) studied the relation between MRV and tail dependence functions. Joe et al. (2010) further studied tail dependence functions and their applications on vine copulas, including the most detailed properties of tail dependence functions. In order to quantify the strength of dependence in the tails for asymptotic independence structures, Hua

\footnotetext{
Received 3 January 2013; revision received 8 March 2013.

* Postal address: Division of Statistics, Northern Illinois University, DeKalb, IL, 60115, USA.

Email address: hua@math.niu.edu

Supported by a start-up grant at Northern Illinois University.

** Postal address: Department of Statistics, University of British Columbia, Vancouver, BC, V6T1Z4, Canada.

Email address: harry.joe@ubc.ca

Supported by an NSERC Canada Discovery grant.

*** Postal address: Department of Mathematics, Washington State University, Pullman, WA, 99164, USA.

Email address: lih@math.wsu.edu

Supported by NSF grants CMMI 0825960 and DMS 1007556.
} 
and Joe (2011) introduced the concept of tail orders for copulas. The tail order corresponds to the reciprocal of the tail coefficient $\eta$ introduced in Ledford and Tawn (1996). The $\eta$ is referred to as the residual dependence index in Hashorva (2010), and the extreme residual coefficient in de Haan and Zhou (2011). With the MRV or HRV methods, univariate marginal distributions are usually transformed to Pareto-like distributions, whereas with the copula method, univariate marginal distributions are transformed to the standard uniform distribution over $[0,1]$. In this paper, we aim to find the relation between these two approaches.

To explain the two approaches, the following notation will be used throughout the paper. Let

$$
I_{d}:=\{1, \ldots, d\}, \quad \overline{\mathbb{R}}_{+}^{d}:=[0, \infty]^{d}, \quad \text { and } \quad \mathbb{R}_{+}^{d}:=[0, \infty)^{d} .
$$

For any $d$-dimensional real vector $\boldsymbol{x}, x_{[i]}$ denotes the $i$ th largest component, and $x_{(i)}$ denotes the $i$ th smallest component. For a subset $I \subseteq I_{d},|I|$ is the cardinality of $I ; \boldsymbol{x}_{I}:=\left(x_{i}, i \in I\right)$. If $\left(x_{j}, j \in J\right), J \subseteq I_{d}$, is a collection of the variables $x_{j}$ then $\left(x_{J}\right)_{[i]}$ is the $i$ th largest of these $x_{j}$, and, similarly, $\left(x_{J}\right)_{(i)}$ is the $i$ th smallest of these $x_{j}$. For any two vectors $\boldsymbol{x}, \boldsymbol{y} \in \mathbb{R}^{d}$, the sum $\boldsymbol{x}+\boldsymbol{y}$, product $\boldsymbol{x} \boldsymbol{y}$, quotient $\boldsymbol{x} / \boldsymbol{y}$, and vector power and vector inequalities such as $\boldsymbol{x} \leq \boldsymbol{y}$ are all operated componentwise.

For a measurable function $g: \mathbb{R}_{+} \rightarrow \mathbb{R}_{+}$, if, for any $t>0, \lim _{x \rightarrow \infty} g(x t) / g(x)=t^{\alpha}$ with $\alpha \in \mathbb{R}$, then $g$ is said to be regularly varying at $\infty$ with variation exponent $\alpha$, and we denote this as $g \in \mathrm{RV}_{\alpha}$ (written as $g \in \mathrm{RV}_{\alpha}(0)$ if $g$ is regularly varying at 0 ); if $\alpha=0$ then $g$ is said to be slowly varying at $\infty$ and we often specifically use $\ell$ as a slowly varying function. For two positive functions $g$ and $h, g(t) \sim h(t)$ as $t \rightarrow t_{0}$ means that $\lim _{t \rightarrow t_{0}}[g(t) / h(t)]=1$. For $l, m \in I_{d}, E^{(l)}:=\left\{\boldsymbol{x} \in \overline{\mathbb{R}}_{+}^{d}: x_{[l]}>0\right\}$ and, thus, $E^{(m)} \subseteq E^{(l)}$ if $l \leq m$. Also, define $E_{0}^{(l)}:=\left\{\boldsymbol{x} \in \mathbb{R}_{+}^{d}: x_{[l]}>0\right\}$. Let $\nu_{l}(\cdot)$ denote the corresponding limit measure (also known as the intensity measure) for MRV on the subcone $E^{(l)}$. In what follows, we use $-\alpha_{l}$ with $\alpha_{l}>0$ as the corresponding exponent of regular variation on $E^{(l)}$ and we call $\alpha_{l}>0$ its tail index.

Let $\boldsymbol{X}$ be a random vector with identical univariate margins $F_{i}, 1 \leq i \leq d$, which are regularly varying with tail index $\alpha>0$ (i.e. $\bar{F}_{i} \in \mathrm{RV}_{-\alpha}$ ). Roughly speaking, if the decay rate of certain joint tail probabilities of $\boldsymbol{X}$ is comparable to the tail decay rate of the marginal survival function $1-F_{i}$, then the usual tail dependence (see, e.g. Joe (1997, p. 33)) appears. However, if the dependence in the upper tail is not sufficiently strong then the joint tail probability decays at a faster rate that may be comparable to that of a function $g \in \mathrm{RV}_{-\alpha_{2}}$ with a larger tail index $\alpha_{2}>\alpha$, and so we need to use HRV to capture the dependence structure hidden in the upper tail interior. Alternatively, we can use a copula $C$ to capture the dependence structure of $\boldsymbol{X}$. In this paper, in order to avoid cumbersome technical arguments on copulas, we assume that the univariate marginal distribution functions, the $F_{i}$ s, are all continuous, so that the copula $C$ is uniquely determined (see Sklar (1959)). However, note that the results on the asymptotic analysis of copulas do not rely on the continuity assumption of margins. Let $\widehat{C}$ be the corresponding survival copula of $\boldsymbol{X}$. If $\widehat{C}(u, \ldots, u) \sim u^{\kappa} \ell(u)$ as $u \rightarrow 0^{+}$then $\kappa$ is referred to as the upper tail order of $C$ and $\kappa \geq 1$ (see Hua and Joe (2011)). Here, a larger $\kappa$ tends to lead to a weaker dependence in the upper tail. In this paper we will show that, for the HRV case, $\kappa=\alpha_{2} / \alpha$. We will study the relation between the intensity measures of MRV or HRV and the tail dependence/order functions of copulas; moreover, through defining an upper exponent function on a subcone for copulas, we will explicitly obtain the expression that connects the HRV intensity measure and tail order functions.

Recently, Mitra and Resnick (2010), (2011) employed order statistics to construct a productmeasure decomposition for characterizing HRV. Their approach overcomes the issue of infinite angular measures for HRV, studied earlier in Maulik and Resnick (2004) or Section 9.4.1 of 
Resnick (2007). We find that the finite-directional measure studied in Mitra and Resnick (2010), (2011) can also be used to characterize the upper exponent functions for copulas. Furthermore, a mixture model for HRV is studied in which the distribution function of a mixing random vector will be related to the finite-directional measure in the product-measure decomposition for HRV.

The remainder of this paper is organized as follows. In Section 2 the relation between MRV and the usual tail dependence or the upper tail order $\kappa=1$ will be discussed. In Section 3 the relation will be extended to the comparison between HRV and the upper tail order $\kappa$. The relation between the tail order function and a Pickands-type representation with finitedirectional measure for HRV will be investigated in Section 4. We present a mixture model for HRV in Section 5, and, finally, in Section 6 we provide some remarks to conclude the paper.

\section{Tail dependence for MRV}

Let $\boldsymbol{X}:=\left(X_{1}, \ldots, X_{d}\right)$ be a random vector with joint cumulative distribution function (CDF) $F$ and continuous univariate margins $F_{1}, \ldots, F_{d}$. Without loss of generality, we may assume that $\boldsymbol{X}$ is nonnegative componentwise. Consider the standard case in which the survival functions $\bar{F}_{i}(x):=1-F_{i}(x), 1 \leq i \leq d$, of the univariate margins are right tail equivalent, that is,

$$
\frac{\bar{F}_{i}(x)}{\bar{F}_{1}(x)}=\frac{1-F_{i}(x)}{1-F_{1}(x)} \rightarrow 1 \quad \text { as } x \rightarrow \infty, 1 \leq i \leq d .
$$

The distribution $F$ or the random vector $X$ is said to be of MRV at $\infty$ with intensity measure $v$ if there exists a scaling function $h(t) \uparrow \infty$ and a nonzero Radon measure $v(\cdot)$ such that, as $t \uparrow \infty$, the following vague convergence holds:

$$
t \mathbb{P}\left(\frac{\boldsymbol{X}}{h(t)} \in \cdot\right) \stackrel{v}{\rightarrow} v(\cdot) \quad \text { in cone } \overline{\mathbb{R}}_{+}^{d} \backslash\{\mathbf{0}\}
$$

that is, $t \mathbb{P}(\boldsymbol{X} / h(t) \in B) \rightarrow v(B)$ for any relatively compact set $B \subseteq \overline{\mathbb{R}}_{+}^{d} \backslash\{\boldsymbol{0}\}$, with $v(\partial B)=0$. Note that, the MRV discussed in this section is actually the MRV on the cone $E^{(1)}$, and MRV can also be defined on subcones in the sense of (3.1) below. The extremal dependence information of $\boldsymbol{X}$ is encoded in the intensity measure $v$ that satisfies $v(t B)=t^{-\alpha} v(B)$ for all relatively compact subsets $B$ that are bounded away from the origin, where $\alpha>0$ is known as the tail index for MRV (i.e. $-\alpha$ is the exponent of variation for MRV). Since the set $B_{1}=\left\{x \in \overline{\mathbb{R}}_{+}^{d}: x_{1}>1\right\}$ is relatively compact within the cone $\overline{\mathbb{R}}_{+}^{d} \backslash\{\mathbf{0}\}$ with $v\left(\partial B_{1}\right)=0$ and $v\left(B_{1}\right)>0$ under (2.1), it follows from (2.2) that the scaling function $h(t)$ can be chosen to satisfy $\bar{F}_{1}(h(t))=t^{-1}, t>0$, after appropriately normalizing the intensity measure by $v\left(B_{1}\right)$. That is, $h(t)$ can be chosen as $h(t)=\bar{F}_{1}^{\leftarrow}\left(t^{-1}\right)=F_{1}^{\leftarrow}\left(1-t^{-1}\right)$ under condition $(2.1)$, where $F_{i}^{\leftarrow}(t):=\inf \left(x: F_{i}(x) \geq t\right)$ is the quantile function of $F_{i}$, and $F_{i}^{\leftarrow}$ is left continuous. Therefore, (2.2) can be equivalently expressed as

$$
\lim _{t \rightarrow \infty} \frac{\mathbb{P}(\boldsymbol{X} \in t B)}{\mathbb{P}\left(X_{1}>t\right)}=v(B) \quad \text { for all relatively compact sets } B \subseteq \overline{\mathbb{R}}_{+}^{d} \backslash\{\boldsymbol{0}\},
$$

satisfying $v(\partial B)=0$. It follows from (2.3) and (2.1) that, for $1 \leq i \leq d$,

$$
\lim _{t \rightarrow \infty} \frac{\mathbb{P}\left(X_{i}>t s\right)}{\mathbb{P}\left(X_{i}>t\right)}=v\left((s, \infty] \times \overline{\mathbb{R}}_{+}^{d-1}\right)=s^{-\alpha} v\left((1, \infty] \times \overline{\mathbb{R}}_{+}^{d-1}\right) \quad \text { for all } s>0
$$


That is, univariate margins have regularly varying right tails and $\bar{F}_{i} \in \mathrm{RV}_{-\alpha}$. In general, a Borel-measurable function $g: \mathbb{R}_{+} \mapsto \mathbb{R}_{+}$is regularly varying with exponent $\rho \in \mathbb{R}$, denoted as $g \in \mathrm{RV}_{\rho}$, if and only if

$$
g(t)=t^{\rho} \ell(t) \quad \text { with } \ell(\cdot) \geq 0 \text { satisfying } \lim _{t \rightarrow \infty} \frac{\ell(t s)}{\ell(t)}=1 \text { for } s>0 .
$$

The function $\ell(\cdot)$ is slowly varying, that is, $\ell \in \mathrm{RV}_{0}$. Since $\bar{F}_{1} \in \mathrm{RV}_{-\alpha}, 1 / \bar{F}_{1} \in \mathrm{RV}_{\alpha}$, by Proposition 2.6(v) of Resnick (2007), the scaling function $h$ in (2.2) satisfies $h \in \mathrm{RV}_{\alpha^{-1}}$. Since all the margins are tail equivalent, as assumed in (2.1), we have

$$
\bar{F}_{i}(t)=t^{-\alpha} \ell_{i}(t), \quad \text { where } \ell_{i} \in \mathrm{RV}_{0} \text { and } \frac{\ell_{i}(t)}{\ell_{j}(t)} \rightarrow 1 \text { as } t \rightarrow \infty \text { for any } i \neq j,
$$

which, together with $\bar{F}_{1}(h(t))=t^{-1}$, implies that

$$
\lim _{t \rightarrow \infty} t \mathbb{P}\left(X_{i}>h(t) s\right)=\frac{\mathbb{P}\left(X_{i}>h(t) s\right)}{\bar{F}_{i}(h(t))} \frac{\bar{F}_{i}(h(t))}{\bar{F}_{1}(h(t))}=s^{-\alpha}, \quad s>0,1 \leq i \leq d .
$$

More detailed discussions on univariate regular variation and MRV can be found in Bingham et al. (1987) and Resnick (1987), (2007). The extension of MRV beyond the nonnegative orthant can be done using the tail probability of $\|\boldsymbol{X}\|$, where $\|\cdot\|$ denotes a norm on $\mathbb{R}^{d}$, in place of the marginal tail probability in (2.3) (see Resnick (2007, Section 6.5.5)). The case in which the limit in (2.1) is any nonzero constant can be easily converted into the standard tail-equivalent case by properly rescaling margins. If the limit in (2.1) is 0 or $\infty$ then some margins have heavier tails than others. One way to overcome this problem is to standardize the margins via marginal monotone transforms.

A copula $C$ is a multivariate distribution with standard uniformly distributed margins on $[0,1]$. Sklar's theorem (see, e.g. Joe (1997, Section 1.6)) states that every multivariate distribution $F$ with univariate margins $F_{1}, \ldots, F_{d}$ can be written as $F\left(x_{1}, \ldots, x_{d}\right)=$ $C\left(F_{1}\left(x_{1}\right), \ldots, F_{d}\left(x_{d}\right)\right)$ for some $d$-dimensional copula $C$. In the case of continuous univariate CDFs, $C$ is unique and

$$
C\left(u_{1}, \ldots, u_{d}\right)=F\left(F_{1}^{\leftarrow}\left(u_{1}\right), \ldots, F_{d}^{\leftarrow}\left(u_{d}\right)\right)
$$

Let $\left(U_{1}, \ldots, U_{d}\right)$ denote a random vector with $U_{i}, 1 \leq i \leq d$, being uniformly distributed on $[0,1]$. The survival copula $\widehat{C}$ is defined as

$$
\widehat{C}\left(u_{1}, \ldots, u_{n}\right)=\mathbb{P}\left(1-U_{1} \leq u_{1}, \ldots, 1-U_{n} \leq u_{n}\right)=\bar{C}\left(1-u_{1}, \ldots, 1-u_{n}\right),
$$

where $\bar{C}:=1+\sum_{\varnothing \neq I \subset I_{d}}(-1)^{|I|} C_{I}$, is the joint survival function of $C$, where $C_{I}$ is the copula for the $I$-margin. The lower and upper tail dependence functions, introduced in Jaworski (2006), Klüppelberg et al. (2008), Nikoloulopoulos et al. (2009), and Joe et al. (2010), are defined as

$$
\begin{aligned}
b^{\mathrm{L}}(\boldsymbol{w} ; C) & :=\lim _{u \rightarrow 0^{+}} \frac{C\left(u w_{i}, 1 \leq i \leq d\right)}{u} \quad \text { for all } \boldsymbol{w}>\mathbf{0}, \\
b^{\mathrm{U}}(\boldsymbol{w} ; C) & :=\lim _{u \rightarrow 0^{+}} \frac{\bar{C}\left(1-u w_{i}, 1 \leq i \leq d\right)}{u} \quad \text { for all } \boldsymbol{w}>\mathbf{0},
\end{aligned}
$$

provided the limits exist. Since $b^{\mathrm{L}}(\boldsymbol{w} ; \widehat{C})=b^{\mathrm{U}}(\boldsymbol{w} ; C)$, a result on upper tail dependence can be easily translated into a similar result for lower tail dependence, and, thus, we only focus on 
upper tail dependence in this paper. Instead of upper orthants used in (2.8), it is often more convenient to work with the complements of lower orthants, leading to the upper exponent function (see Nikoloulopoulos et al. (2009) and Joe et al. (2010)), that is,

$$
a^{\mathrm{U}}(\boldsymbol{w} ; C):=\lim _{u \rightarrow 0^{+}} \frac{\mathbb{P}\left(\bigcup_{i=1}^{d}\left\{U_{i}>1-u w_{i}\right\}\right)}{u}, \quad \boldsymbol{w} \in E_{0}^{(1)},
$$

provided the limits exist. Note that exponent functions and tail dependence functions are related through inclusion-exclusion relations. If the exponent function $a^{\mathrm{U}}(\cdot ; C)$ exists for a $d$-dimensional copula $C$ then the exponent function $a^{\mathrm{U}}\left(\boldsymbol{w}_{I} ; C_{I}\right)$ of any multivariate margin $C_{I}\left(u_{i}, i \in I\right)$ of $C$ also exists. Therefore, the existence of the exponent function $a^{\mathrm{U}}(\cdot ; C)$ implies that the upper tail dependence function $b^{\mathrm{U}}\left(\cdot ; C_{I}\right)$ of any multivariate margin $C_{I}\left(u_{i}, i \in I\right)$ of $C$ exists (may be 0$)$.

With the copula approach, the intensity measure $v$ in (2.3) can be decomposed, as was shown in Li and Sun (2009), into the scale-invariant tail dependence and tail index. For example, let $X_{1}, \ldots, X_{d}$ be nonnegative random variables with survival functions $\bar{F}_{i}$ satisfying (2.1) and $\bar{F}_{1} \in \mathrm{RV}_{-\alpha}$. Suppose that $X:=\left(X_{1}, \ldots, X_{d}\right)$ is upper tail comonotonic (see Hua and Joe (2012a), (2012b)) with copula $C$, that is, the upper tail dependence function is $b^{\mathrm{U}}\left(w_{1}, \ldots w_{d}\right)=\lim _{u \rightarrow 0^{+}} \bar{C}\left(1-u w_{1}, \ldots, 1-u w_{d}\right) / u=\min \left\{w_{1}, \ldots, w_{d}\right\}$ for $w_{i}>$ $0, i=1, \ldots, d$. By definition, $a^{\mathrm{U}}(\boldsymbol{w} ; C)=\sum_{\varnothing \neq I \subseteq I_{d}}(-1)^{|I|-1} b\left(\boldsymbol{w}_{I} ; C_{I}\right)$. By Proposition 2 of Hua and Joe $(2012 \mathrm{~b}), b\left(\boldsymbol{w}_{I} ; C_{I}\right)=\min \left\{w_{i}, i \in \in I\right\}$. Without loss of generality, let $w_{1} \leq \cdots \leq w_{d}$. Then $a^{\mathrm{U}}(\boldsymbol{w} ; C)=\sum_{j=0}^{d-1} \sum_{i=1}^{d-j}(-1)^{j} w_{i}\left(\begin{array}{c}d-i \\ j\end{array}\right)=w_{d}=\max \left\{w_{1}, \ldots, w_{d}\right\}$. Since upper tail comonotonicity corresponds to asymptotic full dependence, by Resnick (2007, p. 196), the intensity measure $v$ satisfies $v\left([\mathbf{0}, \mathbf{x}]^{c}\right)=\left(\min \left\{x_{1}, \ldots, x_{d}\right\}\right)^{-\alpha}$. Then, by Theorem 2.3 of Li and Sun (2009), we shall have $a^{\mathrm{U}}(\boldsymbol{w} ; C)=\left(\min \left\{w_{1}^{-1 / \alpha}, \ldots, w_{d}^{-1 / \alpha}\right\}\right)^{-\alpha}=$ $\max \left\{w_{1}, \ldots, w_{d}\right\}$, which is consistent with what we have derived.

\section{Tail order for HRV on subcones}

The regular variation property (2.3) defined on the cone $\overline{\mathbb{R}}_{+}^{d} \backslash\{\boldsymbol{0}\}$ employs the relatively faster scaling $h(t)$ that is necessary for convergence on the margins (see (2.6)), but such a coarse normalization fails to reveal the finer dependence structure that may be present in the interior. A scaling of smaller order is necessary for any regular variation properties resided or hidden in a smaller cone $E^{(l)}$ for $l=2,3, \ldots, d$. Precisely speaking, the MRV discussed in Section 2 is the MRV on the cone $E^{(1)}$. MRV can also be defined on subcones (see Mitra and Resnick (2010), (2011)). Let $\boldsymbol{X}$ be a nonnegative random vector. Then $\boldsymbol{X}$ possesses MRV on a subcone $E^{(l)}$ if there exists a scaling function $h_{l}(t) \uparrow \infty$ and a nonzero Radon measure $\nu_{l}$ such that

$$
t \mathbb{P}\left(\frac{X}{h_{l}(t)} \in \cdot\right) \stackrel{v}{\rightarrow} v_{l}(\cdot) \quad \text { in } E^{(l)}, \text { as } t \rightarrow \infty
$$

Moreover, if $\boldsymbol{X}$ also has MRV on a subcone $E^{(m)}$ that is a proper subset of $E^{(l)}$ then $\boldsymbol{X}$ is said to possess HRV on $E^{(m)}$.

In order to illustrate the relation between the tail order of a copula and HRV, we first focus on the simpler case where HRV is defined on the subcone $E^{(2)}$. A random vector $\boldsymbol{X}$ is said to have HRV on $E^{(2)}$ if, in addition to (2.2), there exists an increasing scaling function $h_{2}(t) \uparrow \infty$ such that $h(t) / h_{2}(t) \rightarrow \infty$ as $t \rightarrow \infty$, and there exists a nonzero Radon measure $v_{2}$ on $E^{(2)}$ 
such that

$$
t \mathbb{P}\left(\frac{X}{h_{2}(t)} \in B\right) \rightarrow v_{2}(B) \quad \text { as } t \rightarrow \infty
$$

for all relatively compact sets $B \subseteq E^{(2)}$ satisfying $\nu_{2}(\partial B)=0$. See Section 9.4 of Resnick (2007) for more details on HRV on $E^{(2)}$.

Note that $\nu_{2}$ is necessarily homogeneous of order $-\alpha_{2}$ on $E^{(2)}$ for some $\alpha_{2} \geq \alpha$. Consider the set $B_{\wedge}=\left\{\boldsymbol{x} \in \mathbb{R}_{+}^{d}: \bigwedge_{i=1}^{d} x_{i}>1\right\}$, which is relatively compact within $E^{(2)}$. Since $v_{2}(\cdot)$ is nonzero and homogeneous, we must have $\nu_{2}\left(B_{\wedge}\right)>0$. Since (3.2) implies that $t \mathbb{P}\left(\bigwedge_{i=1}^{d} X_{i}>h_{2}(t)\right) \rightarrow v_{2}\left(B_{\wedge}\right)$, we have

$$
\frac{\mathbb{P}\left(\bigwedge_{i=1}^{d} X_{i}>h_{2}(t) s\right)}{\mathbb{P}\left(\bigwedge_{i=1}^{d} X_{i}>h_{2}(t)\right)} \rightarrow \frac{\nu_{2}\left(s B_{\wedge}\right)}{\nu_{2}\left(B_{\wedge}\right)}=s^{-\alpha_{2}} \quad \text { as } t \rightarrow \infty .
$$

Let $\bar{F}_{\wedge}(t):=\mathbb{P}\left(\bigwedge_{i=1}^{d} X_{i}>t\right)$. Then the above limit shows that $\bar{F}_{\wedge} \in \mathrm{RV}_{-\alpha_{2}}$. The scaling function $h_{2}(\cdot)$ can be chosen to satisfy $\bar{F}_{\wedge}\left(h_{2}(t)\right)=t^{-1}, t>0$, after appropriately normalizing the hidden intensity measure by $v_{2}\left(B_{\wedge}\right)$. That is, $h_{2}(t)$ can be chosen as $h_{2}(t)=F_{\wedge}^{\leftarrow}\left(1-t^{-1}\right)$, where $F_{\wedge}^{\leftarrow}(\cdot)$ denotes the left-continuous inverse of $F_{\wedge}(\cdot)$, and, thus, $h_{2} \in \mathrm{RV}_{\alpha_{2}^{-1}}$

Remark 3.1. 1. The typical relatively compact sets in $E^{(2)}$ include subsets $\left\{x_{i}>h_{2}(t) w_{i}, x_{j}>\right.$ $\left.h_{2}(t) w_{j}\right\}, w_{i}>0, w_{j}>0, i \neq j$, whereas the topology on $E^{(1)}$ makes marginal events such as $\left\{x_{i}>h(t) w_{i}\right\}$ relatively compact. In order for $\mathbb{P}\left(X_{i}>h_{2}(t) w_{i}, X_{j}>h_{2}(t) w_{j}\right)$ and $\mathbb{P}\left(X_{i}>h(t) w_{i}\right)$ to decay to 0 at a comparable speed, $h(t)$ has to grow relatively faster than $h_{2}(t)$ does to accommodate the relatively large marginal tail probability. Moreover, if both (2.2) and (3.2) hold, then, for any $w>0$ and $B:=(w, \infty]^{2} \times \overline{\mathbb{R}}_{+}^{d-2} \subseteq E^{(2)}$,

$$
\begin{aligned}
v(B) & =\lim _{t \rightarrow \infty} t \mathbb{P}(\boldsymbol{X} \in h(t) B) \\
& =\lim _{t \rightarrow \infty} t \mathbb{P}\left(\frac{X}{h_{2}(t)} \in \frac{h(t)}{h_{2}(t)} B\right) \\
& \sim v_{2}\left(\frac{h(t)}{h_{2}(t)} B\right) \\
& \rightarrow 0 \quad \text { as } t \rightarrow \infty,
\end{aligned}
$$

because $h(t) / h_{2}(t) \rightarrow \infty$ implies that $h(t) B / h_{2}(t) \rightarrow \varnothing$ as $t \rightarrow \infty$. Thus, HRV on $E^{(2)}$ implies asymptotic independence in the sense that $v\left(E^{(2)}\right)=0$ (see Proposition 5.27 of Resnick (1987) and Property 9.1 of Resnick (2007)).

2. If $F$ has HRV on $E^{(2)}$ then the univariate margins $F_{i}, 1 \leq i \leq d$, have regularly varying right tails with tail index $\alpha$. In contrast, $F$ could have lighter multivariate tails in the interior with tail index $\alpha_{2}$. Geometrically, if $\alpha=\alpha_{2}$ with $h(t) / h_{2}(t) \rightarrow s>0$ ( $s$ is a constant), marginal tails are in comparable magnitude with tails in the interior that link the marginal tails together, resulting in dependence among multivariate extremes. If $\alpha<\alpha_{2}$, tails in the interior are lighter and decay faster than marginal tails, resulting in a lack of tail dependence among random variables. In such a case, scaling that is comparable to lighter tails in the interior must be used to reveal the extremal dependence structure in the interior.

HRV can also be analyzed using the copula method. Since the copula $C$ of distribution $F$ satisfies

$$
\bar{C}\left(1-u w_{i}, 1 \leq i \leq d\right) \leq u \min \left\{w_{1}, \ldots, w_{d}\right\} \quad \text { for all } 0 \leq u \leq 1,\left(w_{1}, \ldots, w_{d}\right) \in \mathbb{R}_{+}^{d},
$$


if the decay rate of the left-hand side with respect to $u$ is faster than $u$ as $u \rightarrow 0$, then the usual tail dependence function would be 0 (i.e. $b^{\mathrm{U}}(\boldsymbol{w}, C) \equiv 0$ ), and in this case a higherorder approximation with scaling $u^{\kappa}, \kappa>1$, must be used to reveal finer information about extremal dependence. Considering this, an upper tail order function (see Hua and Joe (2011)) is introduced, i.e.

$$
b^{\mathrm{U}}(\boldsymbol{w} ; \kappa):=\lim _{u \rightarrow 0^{+}} \frac{\bar{C}\left(1-u w_{i}, 1 \leq i \leq d\right)}{u^{\kappa} \ell(u)}, \quad w_{i}>0,1 \leq i \leq d,
$$

if the limit exists for $\kappa \geq 1$ and some nonnegative function $\ell(u)$ that is slowly varying at 0 (i.e. $\left.\ell\left(t^{-1}\right) \in \mathrm{RV}_{0}\right)$. The lower tail order function can be similarly defined.

The idea of the tail order function is to explore higher-order approximations to extremal dependence in the upper tail from the tail of the uniform margin. If

$$
\bar{C}\left(1-u w_{i}, 1 \leq i \leq d\right) \sim u^{\kappa} \ell(u) b^{\mathrm{U}}(\boldsymbol{w} ; \kappa)=\left[\mathbb{P}\left(F_{i}\left(X_{i}\right)>1-u \ell^{1 / \kappa}(u)\right)\right]^{\kappa} b^{\mathrm{U}}(\boldsymbol{w} ; \kappa),
$$

then, for small $u>0, b^{\mathrm{U}}(\boldsymbol{w} ; \kappa)$ can be used to capture the dependence structure emerged from joint tails at the rate of $\kappa$ times the rate of the marginal tail. The constant $\kappa \geq 1$ is referred to as the tail order. The case where $\kappa=1$ and $\lim _{u \rightarrow 0} \ell(u)=\lambda>0$ corresponds to the usual tail dependence (2.8), and the case where $d>\kappa>1$ may lead to intermediate tail dependence. More precisely, when certain positive dependence assumptions hold (see Hua and Joe (2011)), a copula $C$ is said to have intermediate tail dependence if the limit (3.4) exists and is nonzero for a scaling function $u^{\kappa} \ell(u)$ satisfying

$$
u^{\kappa-1} \ell(u)=\frac{u^{\kappa} \ell(u)}{u} \rightarrow 0 \quad \text { and } \quad u^{\kappa-d} \ell(u)=\frac{u^{\kappa} \ell(u)}{u^{d}} \rightarrow \infty \quad \text { as } u \rightarrow 0 .
$$

That is, the scaling function $u^{\kappa} \ell(u)$ decays to 0 at a faster rate than that of the linear scaling $u$ used in (2.8), but slower than that of $u^{d}$. When $\kappa=d$ and $\ell(u) \rightarrow k$, a finite nonzero value, we refer to this case as tail orthant independence. In this paper we will show that HRV may not only lead to intermediate tail dependence or tail orthant independence, but may also give rise to tail negative dependence (see Remark 4.2 below for a quick impression).

Similar to the situation for a tail dependence function (see Joe et al. (2010)), the existence of the upper tail order function of a copula $C$ does not in general ensure the existence of upper tail order functions of its multivariate margins. Note that the upper tail order function describes the relative decay rate of joint probabilities on upper orthants, whereas, for HRV on different subcones $E^{(l)}, 1 \leq l<d$, the corresponding intensity measure may have masses on complements of lower orthants. That is, upper orthant sets may not contain all compact subsets in $E^{(l)}, 1 \leq l<d$, with an exception of the smallest subcone $E^{(d)}$, in which any compact subset is contained in an upper orthant set. This is a key to establishing the relation between the measure-theoretic MRV method and orthant-based copula approach (see Lemma 6.1 of Resnick (2007)). To this end, we introduce upper exponent functions on subcones. Let $\boldsymbol{U}:=\left(U_{1}, \ldots, U_{d}\right)$ have the copula $C$ with $U_{i}$ being uniformly distributed on $[0,1]$ for each $i$. Define the upper exponent function on $E^{(2)}$ as

$$
a^{\mathrm{U}}(\boldsymbol{w} ; 2, \kappa):=\lim _{u \rightarrow 0^{+}} \frac{\mathbb{P}\left(\bigcup_{i \neq j}\left\{U_{i}>1-u w_{i}, U_{j}>1-u w_{j}\right\}\right)}{u^{\kappa} \ell(u)}, \quad \boldsymbol{w} \in E_{0}^{(2)},
$$

provided the limit exists, where $\ell(u)$ is slowly varying at 0 . Note that the upper exponent function $a^{\mathrm{U}}$ (on $E^{(1)}$ ) in (2.9) describes the tail dependence among univariate margins on 
$[0,1]^{d}$, whereas $a^{\mathrm{U}}(\boldsymbol{w} ; 2, \kappa)$ describes the tail dependence among bivariate margins hidden in $E^{(2)}$. Similarly, we can define the upper exponent function on a subcone $E^{(l)}$ as

$$
a^{\mathrm{U}}(\boldsymbol{w} ; l, \kappa):=\lim _{u \rightarrow 0^{+}} \frac{\mathbb{P}\left(\bigcup_{i_{1} \neq i_{2} \neq \cdots \neq i_{l}}\left\{U_{i_{1}}>1-u w_{i_{1}}, \ldots, U_{i_{l}}>1-u w_{i_{l}}\right\}\right)}{u^{\kappa} \ell(u)}, \quad \boldsymbol{w} \in E_{0}^{(l)},
$$

for $2 \leq l \leq d$, provided the limit exists. Observe that, for $I \subseteq I_{d}$ with $|I|=l, a^{\mathrm{U}}\left(\left(\boldsymbol{w}_{I}, \mathbf{0}_{I^{c}}\right)\right.$; $l, \kappa)=b_{I}^{\mathrm{U}}\left(\boldsymbol{w}_{I} ; \kappa\right)$, the tail order function of the multivariate marginal copula $C_{I}\left(u_{i}, i \in I\right)$.

The upper exponent function on $E^{(2)}$ and upper tail order functions for any multivariate marginal copula $C_{I}$ with $|I| \geq 2$ are related via inclusion-exclusion relations.

Proposition 3.1. If the exponent function $a^{\mathrm{U}}(\boldsymbol{w} ; 2, \kappa)$ defined in (3.6) exists then the upper tail order function $b_{I}^{\mathrm{U}}(\cdot ; \kappa)$ of any multivariate margin $C_{I}$ with $I \subseteq I_{d}$ and $2 \leq|I| \leq d$ also exists.

Proof. We prove the statement by induction. When $d=2, a^{\mathrm{U}}(\cdot ; 2, \kappa)=b^{\mathrm{U}}(\cdot ; \kappa)$. Suppose that the statement is true for dimension $d-1(\geq 1)$ or less. We need to show that the statement is true for dimension $d$. If $a^{\mathrm{U}}(\boldsymbol{w} ; 2, \kappa)$ exists then the upper exponent function $a_{I}^{\mathrm{U}}\left(\boldsymbol{w}_{I} ; 2, \kappa\right)$ for any multivariate margin $C_{I}\left(u_{i}, i \in I\right)$ with $I \subseteq I_{d}$ and $2 \leq|I|<d$ also exists. The induction hypothesis implies that the upper tail order function $b_{I}^{\mathrm{U}}(\cdot ; \kappa)$ of any multivariate margin $C_{I}\left(u_{i}, i \in I\right)$ of $C, I \subset\{1, \ldots, d\},|I| \geq 2$, exists. We now need to show that the tail order function $b^{\mathrm{U}}(w ; \kappa)$ of $C$ exists.

When $d \geq 3$, for $w \in E_{0}^{(2)}$,

$$
\begin{aligned}
& \mathbb{P}\left(\bigcup_{i \neq j}\left\{U_{i}>1-u w_{i}, U_{j}>1-u w_{j}\right\}\right) \\
& \quad=\mathbb{P}\left(\bigcup_{i=1}^{d}\left\{U_{i}>1-u w_{i}\right\}\right)-\sum_{i=1}^{d} \mathbb{P}\left(\left\{U_{i}>1-u w_{i}\right\} \cap\left(\bigcap_{j \neq i}\left\{U_{j} \leq 1-u w_{j}\right\}\right)\right) .
\end{aligned}
$$

For any given $i \in I_{d}$,

$$
\begin{aligned}
\mathbb{P}\left(U_{i}>\right. & \left.1-u w_{i}, U_{j} \leq 1-u w_{j} \text { for all } j \neq i\right) \\
= & \mathbb{P}\left(U_{i}>1-u w_{i}\right)-\mathbb{P}\left(\left\{U_{i}>1-u w_{i}\right\} \cap\left(\bigcup_{j \in I_{d} \backslash\{i\}}\left\{U_{j}>1-u w_{j}\right\}\right)\right) \\
= & \mathbb{P}\left(U_{i}>1-u w_{i}\right)-\sum_{\varnothing \neq J \subseteq I_{d} \backslash\{i\}}(-1)^{|J|-1} \mathbb{P}\left(\bigcap_{j \in J}\left\{U_{i}>1-u w_{i}, U_{j}>1-u w_{j}\right\}\right) \\
= & \mathbb{P}\left(U_{i}>1-u w_{i}\right)-\sum_{j \neq i} \mathbb{P}\left(U_{i}>1-u w_{i}, U_{j}>1-u w_{j}\right)+\cdots \\
& +(-1)^{d-1} \mathbb{P}\left(U_{1}>1-u w_{1}, \ldots, U_{d}>1-u w_{d}\right) .
\end{aligned}
$$

In addition,

$$
\mathbb{P}\left(\bigcup_{i=1}^{d}\left\{U_{i}>1-u w_{i}\right\}\right)=\sum_{\varnothing \neq J \subseteq I_{d}}(-1)^{|J|-1} \mathbb{P}\left(U_{j}>1-u w_{j}, j \in J\right) .
$$


Therefore,

$$
\begin{aligned}
& \mathbb{P}\left(\bigcup_{i \neq j}\left\{U_{i}>1-u w_{i}, U_{j}>1-u w_{j}\right\}\right) \\
& \quad=H\left(u w_{1}, \ldots, u w_{d}\right)-(-1)^{d-1}(d-1) \mathbb{P}\left(U_{1}>1-u w_{1}, \ldots, U_{d}>1-u w_{d}\right),
\end{aligned}
$$

where $H\left(u w_{1}, \ldots, u w_{d}\right)$ is a linear function of $\mathbb{P}\left(U_{j}>1-u w_{j}, j \in I\right), I \subset\{1, \ldots, d\}$, with $d>|I| \geq 2$. As the upper tail order function $b_{I}^{\mathrm{U}}(\cdot ; \kappa)$ of any multivariate margin $C_{I}\left(u_{i}, i \in I\right)$

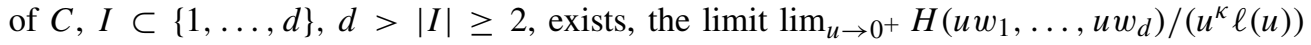
exists. Thus,

$$
b^{\mathrm{U}}(w ; \kappa)=(-1)^{d}(d-1)^{-1}\left[a^{\mathrm{U}}(w ; 2, \kappa)-\lim _{u \rightarrow 0^{+}} \frac{H\left(u w_{1}, \ldots, u w_{d}\right)}{u^{\kappa} \ell(u)}\right]
$$

exists.

Remark 3.2. 1. Similarly, if the upper exponent function $a^{\mathrm{U}}(\boldsymbol{w} ; l, \kappa)$ on $E^{(l)}$ defined in (3.7) exists, then the upper tail order function $b_{I}^{\mathrm{U}}(\cdot ; \kappa)$ of any marginal copula $C_{I}$ with $I \subseteq I_{d}$ and $l \leq|I| \leq d$ also exists. In particular, $a^{\mathrm{U}}(\boldsymbol{w} ; d, \kappa)=b^{\mathrm{U}}(\boldsymbol{w} ; \kappa)$ in the smallest subcone $E^{(d)}$.

2. In general, the upper exponent function $a^{\mathrm{U}}(\boldsymbol{w} ; l, \kappa) \not \equiv 0$ on $E^{(l)}$ if and only if the upper tail order functions $b_{I}^{\mathrm{U}}(\cdot ; \kappa)$ are nonzero for at least one $|I|$-dimensional margin $C_{I}$ with $|I|=l$.

Relations between the tail order and HRV can be established by Propositions 3.2 and 3.3 below. The proofs of the two propositions need repeated use of some operating properties of regularly varying functions. We refer the reader to Proposition 2.6 of Resnick (2007) for references.

The following result establishes the relation between HRV on $E^{(2)}$ and the upper exponent function $a^{\mathrm{U}}(\cdot ; 2, \kappa)$ on $E^{(2)}$, where the $\kappa$ is determined by the tail index $\alpha$ for MRV on $E^{(1)}$ and the tail index $\alpha_{2}$ for MRV on $E^{(2)}$.

Proposition 3.2. Let $\boldsymbol{X}=\left(X_{1}, \ldots, X_{d}\right)$ be a nonnegative random vector with distribution $F$, continuous margins $F_{1}, \ldots, F_{d}$ satisfying (2.1), and copula $C$. Assume that $F$ is regularly varying with intensity measure $v$ and tail index $\alpha$. If $F$ has $H R V$ on $E^{(2)}$ with intensity measures $\nu_{2}$ and tail index $\alpha_{2}$, then the upper exponent function $a^{\mathrm{U}}(\boldsymbol{w} ; 2, \kappa)$ of $C$ exists, where $\kappa=\alpha_{2} / \alpha$ and

$$
a^{\mathrm{U}}(\boldsymbol{w} ; 2, \kappa)=v_{2}\left(\left\{\boldsymbol{x} \geq \mathbf{0}:\left(\boldsymbol{w}^{1 / \alpha} \boldsymbol{x}\right)_{[2]}>1\right\}\right) \text { for all continuity points } \boldsymbol{w} \in E^{(2)} .
$$

Thus, the upper tail order function $b_{I}^{\mathrm{U}}(\cdot ; \kappa)$ for $C_{I}$ exists, where $C_{I}$ is the copula for the I-margin with $2 \leq|I| \leq d$, and, in particular, $b^{\mathrm{U}}\left(w_{1}, w_{2} ; \kappa\right)=v_{2}\left(\left(w_{1}^{-1 / \alpha}, \infty\right] \times\left(w_{2}^{-1 / \alpha}, \infty\right]\right)$.

Proof. HRV on $E^{(2)}$ implies that there exists a function $h_{2} \in \mathrm{RV}_{\alpha_{2}^{-1}}$ that satisfies (3.2). Then the left-continuous inverse $h_{2} \leftarrow(\cdot) \in \mathrm{RV}_{\alpha_{2}}$, and, thus, the reciprocal of the inverse can be written as $\left[h_{2} \leftarrow(t)\right]^{-1}=t^{-\alpha_{2}} \ell_{0}(t)$ for some $\ell_{0} \in \mathrm{RV}_{0}$. Combining this expression with (3.2) yields

$$
\frac{\mathbb{P}(\boldsymbol{X} \in t B)}{\left[h_{2} \leftarrow(t)\right]^{-1}}=\frac{\mathbb{P}(\boldsymbol{X} \in t B)}{t^{-\alpha_{2} \ell_{0}(t)}} \rightarrow v_{2}(B) \quad \text { as } t \rightarrow \infty
$$

for all relatively compact sets $B \subseteq E^{(2)}$ satisfying $\nu_{2}(\partial B)=0$. 
Since $\bar{F}_{1} \in \mathrm{RV}_{-\alpha}, \bar{F}_{1}^{\leftarrow}(u)$ is regularly varying at 0 with exponent $-1 / \alpha$. That is,

$$
t_{u}:=\bar{F}_{1}^{\leftarrow}(u)=u^{-1 / \alpha} \ell_{1}(u)
$$

for some function $\ell_{1}(\cdot)$ that is slowly varying at 0 . Note that $u \rightarrow 0$ if and only if $t_{u} \rightarrow \infty$.

Let $\ell(u):=\left[\ell_{1}(u)\right]^{-\alpha_{2}} \ell_{0}\left(u^{-1 / \alpha} \ell_{1}(u)\right)$. It follows from Proposition 2.6(iv) of Resnick (2007) that $\ell_{0}\left(u^{-1 / \alpha} \ell_{1}(u)\right)$ is slowly varying at 0 , and, thus, $\ell(u)$ is slowly varying at 0 . For any fixed $\boldsymbol{w} \in E_{0}^{(2)}, \kappa=\alpha_{2} / \alpha$, consider

$$
\begin{aligned}
& a_{u}^{\mathrm{U}}(\boldsymbol{w} ; 2, \kappa)=\frac{\mathbb{P}\left(\bigcup_{i \neq j}\left\{U_{i}>1-u w_{i}, U_{j}>1-u w_{j}\right\}\right)}{u^{\kappa} \ell(u)} \\
& =\frac{\mathbb{P}\left(\bigcup_{i \neq j}\left\{F_{i}\left(X_{i}\right)>1-w_{i} u, F_{j}\left(X_{j}\right)>1-w_{j} u\right\}\right)}{\left[u^{-1 / \alpha} \ell_{1}(u)\right]^{-\alpha_{2}} \ell_{0}\left(u^{-1 / \alpha} \ell_{1}(u)\right)} \\
& =\frac{\mathbb{P}\left(\bigcup_{i \neq j}\left\{X_{i}>\bar{F}_{i}^{\leftarrow}\left(w_{i} u\right), X_{j}>\bar{F}_{j}^{\leftarrow}\left(w_{j} u\right)\right\}\right)}{t_{u}^{-\alpha_{2}} \ell_{0}\left(t_{u}\right)} .
\end{aligned}
$$

Since the margins are continuous and $\bar{F}_{i}(x) / \bar{F}_{1}(x) \rightarrow 1$ as $x \rightarrow \infty, \bar{F}_{i}^{\leftarrow}(u) / \bar{F}_{1}^{\leftarrow}(u) \rightarrow 1$ as $u \rightarrow 0^{+}$. Since $\bar{F}_{i}(x)=x^{-\alpha} \ell_{i}(x)$ for $x \geq 0, \bar{F}_{i}^{\leftarrow}(u)$ is regularly varying at 0 with exponent $-\alpha^{-1}$, or, more precisely, $\bar{F}_{i}^{\leftarrow}(u s) / \bar{F}_{i}^{\leftarrow}(u) \rightarrow s^{-1 / \alpha}$ as $u \rightarrow 0^{+}$for any $s>0$. Thus, $\bar{F}_{i}^{\leftarrow}\left(w_{i} u\right) / \bar{F}_{1}^{\leftarrow}(u) \rightarrow w_{i}^{-1 / \alpha}$ as $u \rightarrow 0^{+}$, and, for any small $\varepsilon>0$, when $u$ is sufficiently small (see, e.g. Proposition 2.6 of Resnick (2007)),

$$
(1-\varepsilon) w_{i}^{-1 / \alpha}<\frac{\bar{F}_{i}^{\leftarrow}\left(w_{i} u\right)}{\bar{F}_{1}^{\leftarrow}(u)}<(1+\varepsilon) w_{i}^{-1 / \alpha} \quad \text { for any } 1 \leq i \leq d .
$$

Combining these inequalities with (3.9) and (3.10), we have, for any $\varepsilon>0$, when $u$ is sufficiently small,

$$
\begin{aligned}
& \frac{\mathbb{P}\left(\bigcup_{i \neq j}\left\{X_{i}>t_{u} w_{i}^{-1 / \alpha}(1-\varepsilon), X_{j}>t_{u} w_{j}^{-1 / \alpha}(1-\varepsilon)\right\}\right)}{t_{u}^{-\alpha_{2}} \ell_{0}\left(t_{u}\right)} \\
& \quad \geq a_{u}^{\mathrm{U}}(\boldsymbol{w} ; 2, \kappa) \\
& \geq \frac{\mathbb{P}\left(\bigcup_{i \neq j}\left\{X_{i}>t_{u} w_{i}^{-1 / \alpha}(1+\varepsilon), X_{j}>t_{u} w_{j}^{-1 / \alpha}(1+\varepsilon)\right\}\right)}{t_{u}^{-\alpha_{2}} \ell_{0}\left(t_{u}\right)} .
\end{aligned}
$$

When $w_{i}=0, w_{i}^{-1 / \alpha}$ is treated as $\infty$. Let

$$
\begin{aligned}
A & :=\left\{\boldsymbol{x} \geq \mathbf{0}: x_{i}>w_{i}^{-1 / \alpha}, x_{j}>w_{j}^{-1 / \alpha}, \text { for some } i \neq j\right\} \\
& =\left\{\boldsymbol{x} \geq \mathbf{0}:\left(\boldsymbol{w}^{1 / \alpha} \boldsymbol{x}\right)_{[2]}>1\right\}, \quad \boldsymbol{w} \in E^{(2)}
\end{aligned}
$$

which is relatively compact on $E^{(2)}$. Then, for any continuity set $A$ with $\nu_{2}(\partial A)=0,(3.8)$ implies that

$$
\nu_{2}((1+\varepsilon) A) \leq \liminf _{u \rightarrow 0^{+}} a_{u}^{\mathrm{U}}(\boldsymbol{w} ; 2, \kappa) \leq \limsup _{u \rightarrow 0^{+}} a_{u}^{\mathrm{U}}(\boldsymbol{w} ; 2, \kappa) \leq \nu_{2}((1-\varepsilon) A)
$$

for any small $\varepsilon>0$. Since $\nu_{2}$ is homogeneous of order $-\alpha_{2}$, the above chain of inequalities becomes

$$
(1+\varepsilon)^{-\alpha_{2}} \nu_{2}(A) \leq \liminf _{u \rightarrow 0^{+}} a_{u}^{\mathrm{U}}(\boldsymbol{w} ; 2, \kappa) \leq \limsup _{u \rightarrow 0^{+}} a_{u}^{\mathrm{U}}(\boldsymbol{w} ; 2, \kappa) \leq(1-\varepsilon)^{-\alpha_{2}} \nu_{2}(A),
$$


from which it follows, with $\varepsilon \rightarrow 0^{+}$, that $\lim _{u \rightarrow 0^{+}} a_{u}^{\mathrm{U}}(\boldsymbol{w} ; 2, \kappa)$ exists, and

$$
\begin{aligned}
a^{\mathrm{U}}(\boldsymbol{w} ; 2, \kappa) & =\lim _{u \rightarrow 0^{+}} a_{u}^{\mathrm{U}}(\boldsymbol{w} ; 2, \kappa) \\
& =v_{2}(A) \\
& =v_{2}\left(\left\{\boldsymbol{x} \geq \mathbf{0}:\left(\boldsymbol{w}^{1 / \alpha} \boldsymbol{x}\right)_{[2]}>1\right\}\right), \quad \boldsymbol{w} \in E^{(2)},
\end{aligned}
$$

as desired. Then Proposition 3.1 implies that the upper tail order functions $b_{I}^{\mathrm{U}}$ exist for $|I| \geq 2$, which completes the proof.

Proposition 3.3 proves that existence of $a^{\mathrm{U}}(\boldsymbol{w} ; 2, \kappa)$ and asymptotically equivalent regularly varying univariate margins will lead to $\mathrm{HRV}$ on $E^{(2)}$.

Proposition 3.3. Let $\boldsymbol{X}=\left(X_{1}, \ldots, X_{d}\right)$ be a nonnegative random vector with distribution $F$, continuous margins $F_{1}, \ldots, F_{d}$ satisfying (2.1), and copula $C$. Assume that $\bar{F}_{1} \in \mathrm{RV}_{-\alpha}$. If, for some $\kappa \geq 1$, limit (3.6) exists with $u^{\kappa} \ell(u) / u \rightarrow 0$, then the distribution of $\boldsymbol{X}, F\left(x_{1}, \ldots, x_{d}\right)=$ $C\left(F_{1}\left(x_{1}\right), \ldots, F_{d}\left(x_{d}\right)\right)$, has HRV on $E^{(2)}$.

Proof. For any $\boldsymbol{w} \in E_{0}^{(1)} \backslash E_{0}^{(2)}, a^{\mathrm{U}}(\boldsymbol{w} ; C)$ is clearly well defined. For any $\boldsymbol{w} \in E_{0}^{(2)}$, let $A_{1}:=\bigcup_{i=1}^{d}\left\{U_{i}>1-u w_{i}\right\}$ and $A_{2}:=\bigcup_{i \neq j}\left\{U_{i}>1-u w_{i}, U_{j}>1-u w_{j}\right\}$. It follows from the inclusion-exclusion relation that

$$
\mathbb{P}\left(A_{1}\right)=\sum_{i=1}^{d} \mathbb{P}\left(U_{i}>1-u w_{i}\right)-\mathbb{P}\left(A_{2}\right)=u\left(\sum_{i=1}^{d} w_{i}\right)-\mathbb{P}\left(A_{2}\right) .
$$

Then (3.6) together with (3.13) implies that

$$
a^{\mathrm{U}}(\boldsymbol{w} ; C)=\lim _{u \rightarrow 0^{+}} \frac{\mathbb{P}\left(A_{1}\right)}{u}=\sum_{i=1}^{d} w_{i}-\lim _{u \rightarrow 0^{+}} \frac{\mathbb{P}\left(A_{2}\right)}{u^{\kappa} \ell(u)} \frac{u^{\kappa} \ell(u)}{u}=w_{1}+\cdots+w_{d}
$$

exists for any $\boldsymbol{w} \in E_{0}^{(2)}$. Therefore, owing to Theorem 2.3 of Li and Sun (2009), $F$ is MRV on $E^{(1)}$ with tail index $\alpha$, and the corresponding intensity measure $v$ satisfies $v\left(A_{2}\right)=0$ (asymptotic independence), and, moreover, the convergence (i) of Proposition 9.5 of Resnick (2007) follows: for all $z=\left(z_{1}, \ldots, z_{d}\right) \in \mathbb{R}_{+}^{d} \backslash\{\mathbf{0}\}$,

$$
\lim _{t \rightarrow \infty} t \mathbb{P}\left(\max _{1 \leq i \leq d}\left\{z_{i} X_{i}\right\}>h(t) s\right)=s^{-\alpha} a^{\mathrm{U}}\left(z^{\alpha} ; C\right), \quad s>0 .
$$

It follows from Proposition 3.1 that the upper tail order function $b_{I}^{\mathrm{U}}(\cdot ; \kappa)$ of any multivariate margin $C_{I}$ with $I \subseteq I_{d}$ and $2 \leq|I| \leq d$ also exists. For any $\boldsymbol{w} \geq \mathbf{0}, I \subseteq I_{d}$, and $2 \leq|I| \leq d$, consider

$$
b_{u}^{\mathrm{U}}\left(\boldsymbol{w}_{I} ; \kappa\right):=\frac{\mathbb{P}\left(\bigcap_{i \in I}\left\{U_{i}>1-u w_{i}\right\}\right)}{u^{\kappa} \ell(u)}=\frac{\mathbb{P}\left(\bigcap_{i \in I}\left\{X_{i}>\bar{F}_{i}^{\leftarrow}\left(u w_{i}\right)\right\}\right)}{\mathbb{P}\left(X_{1}>\bar{F}_{1}^{\leftarrow}\left(u^{\kappa} \ell(u)\right)\right)},
$$

and assume that $b_{I}^{\mathrm{U}}\left(\boldsymbol{w}_{I} ; \kappa\right)=\lim _{u \rightarrow 0^{+}} b_{u}^{\mathrm{U}}\left(\boldsymbol{w}_{I} ; \kappa\right)$ exists for $\kappa \geq 1$. Here $\bar{F}_{i}^{\leftarrow} \in \mathrm{RV}_{-1 / \alpha}(0)$ implies that, for any small $\varepsilon>0$ and $1 \leq i \leq d$, as $u \rightarrow 0^{+}$,

$$
\frac{\bar{F}_{i}^{\leftarrow}\left((1-\varepsilon) w_{i} u\right)}{\bar{F}_{1}^{\leftarrow}(u)} \rightarrow(1-\varepsilon)^{-1 / \alpha} w_{i}^{-1 / \alpha}, \quad \frac{\bar{F}_{i}^{\leftarrow}\left((1+\varepsilon) w_{i} u\right)}{\bar{F}_{1}^{\leftarrow}(u)} \rightarrow(1+\varepsilon)^{-1 / \alpha} w_{i}^{-1 / \alpha}
$$


For any small $\varepsilon>0$, when $u>0$ is sufficiently small,

$$
\bar{F}_{i}^{\leftarrow}\left((1+\varepsilon) w_{i} u\right) \leq w_{i}^{-1 / \alpha} \bar{F}_{1}^{\leftarrow}(u) \leq \bar{F}_{i}^{\leftarrow}\left((1-\varepsilon) w_{i} u\right), \quad 1 \leq i \leq d,
$$

which, together with (3.14), implies that (when $w_{i}=0, w_{i}^{-1 / \alpha}$ is treated as $\infty$ )

$$
\begin{aligned}
b_{I}^{\mathrm{U}}\left((1-\varepsilon) \boldsymbol{w}_{I} ; \kappa\right) & \leq \liminf _{u \rightarrow 0^{+}} \frac{\mathbb{P}\left(\bigcap_{i \in I}\left\{X_{i}>w_{i}^{-1 / \alpha} \bar{F}_{1}^{\leftarrow}(u)\right\}\right)}{\mathbb{P}\left(X_{1}>\bar{F}_{1}^{\leftarrow}\left(u^{\kappa} \ell(u)\right)\right)} \\
& \leq \limsup _{u \rightarrow 0^{+}} \frac{\mathbb{P}\left(\bigcap_{i \in I}\left\{X_{i}>w_{i}^{-1 / \alpha} \bar{F}_{1}^{\leftarrow}(u)\right\}\right)}{\mathbb{P}\left(X_{1}>\bar{F}_{1}^{\leftarrow}\left(u^{\kappa} \ell(u)\right)\right)} \\
& \leq b_{I}^{\mathrm{U}}\left((1+\varepsilon) \boldsymbol{w}_{I} ; \kappa\right) .
\end{aligned}
$$

Since $b_{I}^{\mathrm{U}}(\cdot ; \kappa)$ is homogeneous of order $\kappa$, the above chain of inequalities becomes

$$
\begin{aligned}
(1-\varepsilon)^{\kappa} b_{I}^{\mathrm{U}}\left(\boldsymbol{w}_{I} ; \kappa\right) & \leq \liminf _{u \rightarrow 0^{+}} \frac{\mathbb{P}\left(\bigcap_{i \in I}\left\{X_{i}>w_{i}^{-1 / \alpha} \bar{F}_{1}^{\leftarrow}(u)\right\}\right)}{\mathbb{P}\left(X_{1}>\bar{F}_{1}^{\leftarrow}\left(u^{\kappa} \ell(u)\right)\right)} \\
& \leq \limsup _{u \rightarrow 0^{+}} \frac{\mathbb{P}\left(\bigcap_{i \in I}\left\{X_{i}>w_{i}^{-1 / \alpha} \bar{F}_{1}^{\leftarrow}(u)\right\}\right)}{\mathbb{P}\left(X_{1}>\bar{F}_{1}^{\leftarrow}\left(u^{\kappa} \ell(u)\right)\right)} \\
& \leq(1+\varepsilon)^{\kappa} b_{I}^{\mathrm{U}}\left(\boldsymbol{w}_{I} ; \kappa\right) .
\end{aligned}
$$

Let $t:=u^{-\kappa}$. As $\varepsilon \rightarrow 0$, the limit

$$
\lim _{t \rightarrow \infty} \frac{\mathbb{P}\left(\bigcap_{i \in I}\left\{X_{i}>w_{i}^{-1 / \alpha} \bar{F}_{1}^{\leftarrow}\left(t^{-1 / \kappa}\right)\right\}\right)}{\mathbb{P}\left(X_{1}>\bar{F}_{1}^{\leftarrow}\left(t^{-1} \ell\left(t^{-1 / \kappa}\right)\right)\right)}=b_{I}^{\mathrm{U}}\left(\boldsymbol{w}_{I} ; \kappa\right)
$$

exists for all $\boldsymbol{w} \geq \mathbf{0}$. Let $g\left(t^{-1}\right):=t^{-1} \ell\left(t^{-1 / \kappa}\right)$, with $g\left(t^{-1}\right)$ eventually decreasing to 0 . Observe that $\ell\left(t^{-1 / \kappa}\right) \in \mathrm{RV}_{0}$, and, thus, we have $g\left(t^{-1}\right) \in \mathrm{RV}_{-1}$. Set $s^{-1}=g\left(t^{-1}\right)$, leading to $t^{-1} \sim g^{\leftarrow}\left(s^{-1}\right)$ as $s \rightarrow \infty$, where $g^{\leftarrow}(\cdot)$ denotes the left-continuous inverse of $g(\cdot)$. Thus, we have

$$
\lim _{s \rightarrow \infty} \frac{s \mathbb{P}\left(\bigcap_{i \in I}\left\{X_{i}>w_{i}^{-1 / \alpha} \bar{F}_{1}^{\leftarrow}\left(\left[g^{\leftarrow}\left(s^{-1}\right)\right]^{1 / \kappa}\right)\right\}\right)}{s \mathbb{P}\left(X_{1}>\bar{F}_{1}^{\leftarrow}\left(s^{-1}\right)\right)}=b_{I}^{\mathrm{U}}\left(\boldsymbol{w}_{I} ; \kappa\right)
$$

Let $h_{2}(s):=\bar{F}_{1}^{\leftarrow}\left(\left[g^{\leftarrow}\left(s^{-1}\right)\right]^{1 / \kappa}\right)$ and $h(s):=\bar{F}_{1}^{\leftarrow}\left(s^{-1}\right)$. Define $\alpha_{2}:=\kappa \alpha \geq \alpha$. Then $h(s) \in$ $\mathrm{RV}_{1 / \alpha}$ and $h_{2}(s) \in \mathrm{RV}_{1 / \alpha_{2}}$. On the other hand, clearly, $s \mathbb{P}\left(X_{1}>h(s)\right) \rightarrow 1$ as $s \rightarrow \infty$. Thus, for $\boldsymbol{w} \geq \mathbf{0}$,

$$
\lim _{t \rightarrow \infty} t \mathbb{P}\left(\frac{X}{h_{2}(t)} \in \prod_{i \in I}\left(w_{i}^{-1}, \infty\right]\right)=b_{I}^{\mathrm{U}}\left(\boldsymbol{w}_{I}^{\alpha} ; \kappa\right) .
$$

Rephrasing this limit differently, we have, for any $I \subseteq I_{d}$ and $2 \leq|I| \leq d$,

$$
\lim _{t \rightarrow \infty} t \mathbb{P}\left(\min _{i \in I}\left\{w_{i} X_{i}\right\}>h_{2}(t) s\right)=b_{I}^{\mathrm{U}}\left(\left(\frac{\boldsymbol{w}_{I}}{s}\right)^{\alpha} ; \kappa\right)=s^{-\alpha_{2}} b_{I}^{\mathrm{U}}\left(\boldsymbol{w}_{I}^{\alpha} ; \kappa\right), \quad s>0,
$$

where $\alpha_{2}=\kappa \alpha$, leading to convergence (ii) of Proposition 9.5 of Resnick (2007), when only components with indexes in $I$ are finite. By Proposition 9.5 of Resnick (2007), $F$ is MRV on $E^{(2)}$. 
Remark 3.3. The proof of Proposition 3.3 also yields an interpretation for the functions $c(\cdot)$ and $d(\cdot)$ in Proposition 9.5 of Resnick (2007) in terms of upper exponent and tail order functions of the underlying copula $C$. That is, if $\kappa=\alpha_{2} / \alpha$ then, for the functions $c(z)$ and $d(z)$,

1. $c(z)=a^{\mathrm{U}}\left(z^{\alpha} ; C\right)$ for all $z \in \mathbb{R}_{+}^{d} \backslash\{0\}$;

2. $d(z)=b_{I}^{\mathrm{U}}\left(z_{I}^{\alpha} ; \kappa\right)$ if only components of $z$ with indexes in $I$ are finite, $I \subseteq I_{d}$, and $2 \leq|I| \leq d$.

For a subspace $E \subseteq \overline{\mathbb{R}}_{+}^{d}$, let $M_{+}(E)$ denote the class of the nonnegative Radon measures on $E$. Mitra and Resnick (2011) provided a representation of MRV on a general subcone $E^{(l)}$ for $l=1, \ldots, d$. In contrast to the previous study on norm-based polar transforms for MRV on subcones, the method in Mitra and Resnick (2011) fixes directions on an order-statisticsbased unit envelope $\delta \mathbb{N}_{l}:=\left\{x \in E^{(l)}: x_{[l]}=1\right\}$ that wraps all open portions of the boundaries of subcone $E^{(l)}$ from within. Note that $\delta \mathbb{N}_{l}$ is always compact within $E^{(l)}$, and this leads to a product-measure representation for the intensity measure $\nu_{l}(\cdot)$ of MRV on $E^{(l)}$, where the spectral or directional measure $S_{l}(\cdot)$ is always finite. We refer the reader to Proposition 3.1 of Mitra and Resnick (2011) for more details.

The order-statistics-based homogeneous transform also yields a representation of Pickands type for the upper exponent function on $E^{(l)}$. The following result illustrates the idea with the subcone $E^{(2)}$.

Proposition 3.4. Let $\boldsymbol{X}=\left(X_{1}, \ldots, X_{d}\right)$ be a nonnegative random vector with distribution $F$ and continuous marginal CDFs $F_{1}, \ldots, F_{d}$ satisfying (2.1). Assume that $F$ has HRV on $E^{(2)}$ with intensity measure $v_{2}$ and tail index $\alpha_{2}$. Then

$$
a^{\mathrm{U}}(\boldsymbol{w} ; 2, \kappa)=\int_{\delta \mathbb{N}_{2}}\left[\left(\boldsymbol{w}^{1 / \alpha} \boldsymbol{s}\right)_{[2]}\right]^{\alpha_{2}} S_{2}(\mathrm{~d} \boldsymbol{s}),
$$

where $\alpha_{2}=\kappa \alpha$ and $S_{2}(\cdot)$ is a finite-directional measure.

Proof. By Proposition 3.2, for any $\boldsymbol{w} \in E^{(2)}$,

$$
a^{\mathrm{U}}(\boldsymbol{w} ; 2, \kappa)=v_{2}\left(\left\{\boldsymbol{x} \geq \mathbf{0}:\left(\boldsymbol{w}^{1 / \alpha} \boldsymbol{x}\right)_{[2]}>1\right\}\right)=: v_{2}(A) .
$$

Noting that $\delta \mathbb{N}_{2}:=\left\{x \in E^{(2)}: x_{[2]}=1\right\}$, let $T: E^{(2)} \rightarrow(0, \infty) \times \delta \mathbb{N}_{2}$ be a transform such that

$$
T(\boldsymbol{y})=\left(y_{[2]}, \frac{\boldsymbol{y}}{y_{[2]}}\right)=:(r, s),
$$

with the left inverse $T^{-1}(r, s)=r s$. Consider

$$
\nu_{2}(A)=v_{2} \circ T^{-1}(T(A))=v_{2} \circ T^{-1}\left(\left\{(r, s): r>\frac{1}{\left(\boldsymbol{w}^{1 / \alpha} \boldsymbol{x}\right)_{[2]}}, s \in \delta \mathbb{N}_{2}\right\}\right) .
$$

By Proposition 3.1 of Mitra and Resnick (2011), there exists a finite measure $S_{2}$ such that $\nu_{2} \circ T^{-1}(\mathrm{~d} r, \mathrm{~d} s)=\alpha_{2} r^{-\alpha_{2}-1} \mathrm{~d} r S_{2}(\mathrm{~d} s)$. Since both measures are finite, we can apply Fubini's theorem to obtain

$$
\nu_{2}(A)=\int_{\delta \mathbb{N}_{2}} \int_{1 /\left(\boldsymbol{w}^{1 / \alpha} \boldsymbol{x}\right)_{[2]}}^{\infty} \alpha_{2} r^{-\alpha_{2}-1} \mathrm{~d} r S_{2}(\mathrm{~d} \boldsymbol{s})=\int_{\delta \mathbb{N}_{2}}\left[\left(\boldsymbol{w}^{1 / \alpha} \boldsymbol{s}\right)_{[2]}\right]^{\alpha_{2}} S_{2}(\mathrm{~d} \boldsymbol{s}),
$$

which completes the proof. 
All of the above results on $E^{(2)}$ can be extended to subcone $E^{(l)}, 3 \leq l \leq d$. For example, if $F$ has HRV on $E^{(l)}$ with intensity measure $v_{l}$ and tail index $\alpha_{l}$, then

$$
a^{\mathrm{U}}(\boldsymbol{w} ; l, \kappa)=\int_{\delta \mathbb{N}_{l}}\left[\left(\boldsymbol{w}^{1 / \alpha} \boldsymbol{s}\right)_{[l]}\right]^{\alpha_{l}} S_{l}(\mathrm{~d} \boldsymbol{s}),
$$

where $\alpha_{l}=\kappa \alpha$ and $S_{l}(\cdot)$ is a finite-directional measure.

\section{Tail order functions}

Tail order functions are directly related to upper exponent functions (see Remark 3.2). Based on representation (3.16), the upper exponent function can be derived from a finite measure $S_{l}$. Since the upper tail order function of a $d$-dimensional copula $C$ coincides with its upper exponent function on $E^{(d)}$, we can construct the upper tail order function from the finite-directional measure $S_{d}$ as well; this will be studied in Section 4.1 with the bivariate case. However, for HRV on $E^{(l)}$ with $1<l<d$, there is an inclusion-exclusion relation between $a^{\mathrm{U}}(\boldsymbol{w} ; l, \kappa)$ and upper tail order functions, and the related discussions and examples will be given in Section 4.2 .

\subsection{Bivariate cases}

When $d=2, a^{\mathrm{U}}\left(w_{1}, w_{2} ; 2, \kappa\right)=b\left(w_{1}, w_{2} ; \kappa\right)$, where $b$ is the upper tail order function that is homogeneous of order $\kappa$. The support of the measure $S_{2}$ is $\left\{\left(s_{2}, 1\right),\left(1, s_{1}\right): 1 \leq s_{1}, s_{2} \leq \infty\right\}$, which is the union of a horizontal line and a vertical line that meet at the corner point $(1,1)$. Let $H_{1}$ be a finite measure defined on the vertical line $[(1,1),(1, \infty)]$, and let $H_{2}$ be a finite measure defined on the horizontal line $[(1,1),(\infty, 1)]$. We can write (since $\left.\kappa=\alpha_{2} / \alpha\right)$

$$
\begin{aligned}
a^{\mathrm{U}}\left(w_{1}, w_{2} ; 2, \kappa\right)= & \int_{(1, \infty]} \min \left\{w_{1}^{\kappa}, s^{\alpha_{2}} w_{2}^{\kappa}\right\} H_{1}(\mathrm{~d} s)+\int_{(1, \infty]} \min \left\{w_{2}^{\kappa}, s^{\alpha_{2}} w_{1}^{\kappa}\right\} H_{2}(\mathrm{~d} s) \\
& +\min \left\{w_{1}^{\kappa}, w_{2}^{\kappa}\right\} S(\{(1,1)\}) .
\end{aligned}
$$

For $w_{1}=1$ and $w_{2}=w>1$,

$$
\begin{aligned}
b(1, w ; \kappa) & =a^{\mathrm{U}}(1, w ; 2, \kappa) \\
& =H_{1}((1, \infty])+\int_{\left(1, w^{1 / \alpha}\right]} s^{\alpha_{2}} H_{2}(\mathrm{~d} s)+w^{\kappa} H_{2}\left(\left(w^{1 / \alpha}, \infty\right]\right)+S(\{(1,1)\}) .
\end{aligned}
$$

If $\mathrm{H}_{2}$ is absolutely continuous with respect to the Lebesgue measure and $h_{2}$ is the density, then $b(1, w ; \kappa)$ is differentiable in $w$, and by Leibniz's rule for integrals,

$$
\frac{\partial b(1, w ; \kappa)}{\partial w}=\kappa w^{\kappa-1} H_{2}\left(\left(w^{1 / \alpha}, \infty\right]\right) .
$$

Therefore, $\mathrm{H}_{2}$ has the representation

$$
H_{2}\left(\left(w^{1 / \alpha}, \infty\right]\right)=\kappa^{-1} w^{1-\kappa} \frac{\partial b(1, w ; \kappa)}{\partial w}=: \kappa^{-1} w^{1-\kappa} g_{2}(w), \quad 1<w \leq \infty .
$$

By symmetry, if $H_{1}$ is absolutely continuous with respect to the Lebesgue measure then

$$
H_{1}\left(\left(w^{1 / \alpha}, \infty\right]\right)=\kappa^{-1} w^{1-\kappa} \frac{\partial b(w, 1 ; \kappa)}{\partial w}=: \kappa^{-1} w^{1-\kappa} g_{1}(w), \quad 1<w \leq \infty .
$$

Now we examine how to relate the tail order function to the strength of dependence. 
Example 4.1. Let $b\left(w_{1}, w_{2} ; \kappa\right)=w_{1}^{\xi} w_{2}^{\kappa-\xi}$, where $1<\kappa<2$ and $0<\xi<\kappa$. It can be an upper tail order function from the survival copula of a bivariate extreme value copula. Then

$$
\begin{array}{cc}
b(1, w ; \kappa)=w^{\kappa-\xi}, & g_{2}(w)=(\kappa-\xi) w^{\kappa-\xi-1}, \\
b(w, 1 ; \kappa)=w^{\xi}, & g_{1}(w)=\xi w^{\xi-1} .
\end{array}
$$

Therefore, by (4.3) and (4.4),

$$
\begin{aligned}
& H_{2}\left(\left(w^{1 / \alpha}, \infty\right]\right)=\kappa^{-1} w^{1-\kappa}(\kappa-\xi) w^{\kappa-\xi-1}=\kappa^{-1}(\kappa-\xi) w^{-\xi}, \\
& H_{1}\left(\left(w^{1 / \alpha}, \infty\right]\right)=\kappa^{-1} w^{1-\kappa} \xi w^{\xi-1}=\kappa^{-1} \xi w^{\xi-\kappa} .
\end{aligned}
$$

Then the densities of the measures $H_{2}$ and $H_{1}$ are

$$
h_{2}(w)=\alpha \xi(\kappa-\xi) \kappa^{-1} w^{-\alpha \xi-1}, \quad h_{1}(w)=\alpha \xi(\kappa-\xi) \kappa^{-1} w^{\alpha(\xi-\kappa)-1},
$$

respectively. Moreover, (4.2) implies that

$$
\begin{aligned}
S(\{(1,1)\}) & =b(1, w ; \kappa)-H_{1}((1, \infty])-\int_{\left(1, w^{1 / \alpha}\right]} s^{\alpha_{2}} H_{2}(\mathrm{~d} s)-w^{\kappa} H_{2}\left(\left(w^{1 / \alpha}, \infty\right]\right) \\
& =w^{\kappa-\xi}-\kappa^{-1} \xi-\kappa^{-1} \xi\left(w^{\kappa-\xi}-1\right)-\kappa^{-1}(\kappa-\xi) w^{\kappa-\xi} \\
& \equiv 0 .
\end{aligned}
$$

Example 4.2. Let $b\left(w_{1}, w_{2} ; \kappa\right)=\left(w_{1}+w_{2}\right)^{\kappa}-w_{1}^{\kappa}-w_{2}^{\kappa}$, where $1<\kappa<2$. It can be an upper tail order function from a bivariate Archimedean copula (see Hua and Joe (2011)). Then

$b(1, w ; \kappa)=b(w, 1 ; \kappa)=(1+w)^{k}-1-w^{\kappa}, \quad g_{1}(w)=g_{2}(w)=\kappa(1+w)^{\kappa-1}-\kappa w^{\kappa-1}$.

Therefore, by (4.3) and (4.4),

$$
\begin{aligned}
H_{1}\left(\left(w^{1 / \alpha}, \infty\right]\right) & =H_{2}\left(\left(w^{1 / \alpha}, \infty\right]\right) \\
& =\kappa^{-1} w^{1-\kappa}\left[\kappa(1+w)^{\kappa-1}-\kappa w^{\kappa-1}\right] \\
& =\left(1+w^{-1}\right)^{\kappa-1}-1,
\end{aligned}
$$

and $h_{1}(w)=h_{2}(w)=\alpha(\kappa-1)\left(w^{-\alpha}+1\right)^{\kappa-2} w^{-\alpha-1}$. Therefore, by (4.2),

$$
\begin{aligned}
S(\{(1,1)\})= & {\left[(1+w)^{k}-1-w^{\kappa}\right]-\left(2^{\kappa-1}-1\right)-\left[(1+w)^{\kappa-1}-2^{\kappa-1}\right] } \\
& -w^{\kappa}\left[\left(1+w^{-1}\right)^{\kappa-1}-1\right] \\
\equiv & 0 .
\end{aligned}
$$

Remark 4.1. Choosing $\xi=\kappa / 2$ in Example 4.1, the densities $h_{1}, h_{2} \in \mathrm{RV}_{-1-\alpha_{2} / 2}$. For Example 4.2, the densities $h_{1}, h_{2} \in \mathrm{RV}_{-1-\alpha}$. Also, $1<\kappa<2$ and $\kappa=\alpha_{2} / \alpha$ imply that $-1-\alpha_{2} / 2>-1-\alpha$. Note that from (4.1), with the same corresponding tail order $\kappa$, the upper tail of a bivariate Archimedean copula may be more dependent than the lower tail of a bivariate extreme value copula in the sense that the tail order function for the Archimedean copula is relatively larger. 
Example 4.3. (Geometric mixtures of comonotonicity and independence.) Let $b\left(w_{1}, w_{2} ; \kappa\right)=$ $\left(w_{1} \wedge w_{2}\right)^{2-\kappa}\left(w_{1} w_{2}\right)^{\kappa-1}, 1<\kappa<2$. This is a geometric mixture of tail order functions of comonotonicity and independence. Then

$$
b(1, w ; \kappa)=b(w, 1 ; \kappa)=w^{\kappa-1}, \quad g_{1}(w)=g_{2}(w)=(\kappa-1) w^{\kappa-2} .
$$

Equations (4.3) and (4.4) imply that

$$
H_{1}\left(\left(w^{1 / \alpha}, \infty\right]\right)=H_{2}\left(\left(w^{1 / \alpha}, \infty\right]\right)=\kappa^{-1} w^{1-\kappa}\left[(\kappa-1) w^{\kappa-2}\right]=\kappa^{-1}(\kappa-1) w^{-1},
$$

and $h_{1}(w)=h_{2}(w)=\alpha \kappa^{-1}(\kappa-1) w^{-\alpha-1}$. Therefore, by (4.2),

$$
\begin{aligned}
S(\{(1,1)\}) & =\left[w^{\kappa-1}\right]-\left[\kappa^{-1}(\kappa-1)\right]-\left[\kappa^{-1}\left(w^{\kappa-1}-1\right)\right]-w^{\kappa}\left[\kappa^{-1}(\kappa-1) w^{-1}\right] \\
& =2 \kappa^{-1}-1 .
\end{aligned}
$$

Given any finite measure on $\delta \mathbb{N}_{2}$ with sufficient regularity conditions (or, equivalently, given that $H_{1}, H_{2}$, and $S(\{(1,1)\})$ are finite, and $H_{1}$ and $H_{2}$ are absolutely continuous), we now study whether the $b$ function based on (4.2) and a parallel expression for $b(w, 1 ; \kappa)$ is always an appropriate tail order function; more specifically, whether

$$
b\left(w_{1}, w_{2} ; \kappa\right)= \begin{cases}w_{1}^{\kappa} b\left(1, \frac{w_{2}}{w_{1}} ; \kappa\right), & 0<w_{1}<w_{2}, \\ w_{2}^{\kappa} b\left(\frac{w_{1}}{w_{2}}, 1 ; \kappa\right), & 0<w_{2} \leq w_{1},\end{cases}
$$

is a tail order function with tail order $\kappa$. Note that, from $(4.1), b(1,1 ; \kappa)$ is consistent for $b(1, w ; \kappa)$ and $b(w, 1 ; \kappa)$, so we can consider expression (4.5). If such a function $b$ is a tail order function for the bivariate case then $b\left(w_{1}, w_{2}\right)$ must be positively homogeneous of order 2 , increasing in each argument, and also 2-increasing. By construction, it is homogeneous of order $\kappa$. It is easy to verify that $b\left(w_{1}, w_{2}\right)$ is increasing in $w_{1}$ and $w_{2}$ by (4.3) and (4.4). Now we check the 2-increasing requirement. Differentiate (4.5) with respect to $w_{1}$ and $w_{2}$. We show the details only for $0<w_{1}<w_{2}$ as the other case is symmetric:

$$
\frac{\partial b\left(w_{1}, w_{2}\right)}{\partial w_{2}}=w_{1}^{\kappa-1} g_{2}\left(\frac{w_{2}}{w_{1}}\right)
$$

and

$$
\frac{\partial^{2} b\left(w_{1}, w_{2}\right)}{\partial w_{1} \partial w_{2}}=(k-1) w_{1}^{\kappa-2} g_{2}\left(\frac{w_{2}}{w_{1}}\right)-w_{2} w_{1}^{\kappa-3} g_{2}^{\prime}\left(\frac{w_{2}}{w_{1}}\right) .
$$

Note that $\partial b\left(w_{1}, w_{2}\right) / \partial w_{2}$ is homogeneous of order $\kappa-1$ and

$$
h\left(w_{1}, w_{2}\right):=\frac{\partial^{2} b\left(w_{1}, w_{2}\right)}{\partial w_{1} \partial w_{2}}
$$

is homogeneous of order $\kappa-2$. Let $w:=w_{2} / w_{1}>1$. Then, without loss of generality,

$$
\begin{aligned}
h\left(w_{1}, w_{2}\right) & =h\left(1, \frac{w_{2}}{w_{1}}\right) w_{1}^{\kappa-2} \\
& =w_{1}^{\kappa-2}\left[(k-1) g_{2}\left(\frac{w_{2}}{w_{1}}\right)-\left(\frac{w_{2}}{w_{1}}\right) g_{2}^{\prime}\left(\frac{w_{2}}{w_{1}}\right)\right] \\
& =w_{1}^{\kappa-2}\left[(k-1) g_{2}(w)-w g_{2}^{\prime}(w)\right] .
\end{aligned}
$$


By (4.3), $g_{2}(w)=\kappa w^{\kappa-1} H_{2}\left(\left(w^{1 / \alpha}, \infty\right]\right)$. Therefore,

$$
\begin{aligned}
(\kappa-1) g_{2}(w)-w g_{2}^{\prime}(w)= & (\kappa-1) \kappa w^{\kappa-1} H_{2}\left(\left(w^{1 / \alpha}, \infty\right]\right)-(\kappa-1) \kappa w^{\kappa-1} H_{2}\left(\left(w^{1 / \alpha}, \infty\right]\right) \\
& +\frac{\kappa}{\alpha} h_{2}\left(w^{1 / \alpha}\right) w^{\kappa+1 / \alpha-1} \\
= & \frac{\kappa}{\alpha} h_{2}\left(w^{1 / \alpha}\right) w^{\kappa+1 / \alpha-1} \\
\geq & 0 .
\end{aligned}
$$

Therefore, $h\left(w_{1}, w_{2}\right) \geq 0$, that is, we have proved that the function $b$ is 2-increasing, and, thus, $b$ is a tail order function.

Moreover, depending on the measures of $H_{1}$ and $H_{2}, b(1, w ; \kappa)$ and $b(w, 1 ; \kappa)$ can be bounded or unbounded as $w \rightarrow \infty$. For example, based on (4.2), if $H_{2}$ is a probability measure defined on $(1, \infty]$ with $F_{H_{2}}(\cdot)$ as the distribution, and $F_{H_{2}} \in \mathrm{RV}_{-\alpha_{2}-\varepsilon}$, then $b(1, w ; \kappa)$ is bounded (unbounded) as $w \rightarrow \infty$ when $\varepsilon>0(\varepsilon<0)$. Examples 4.1, 4.2, and 4.3 have unbounded $b(1, w ; \kappa)$. Given a tail dependence function $b^{\mathrm{U}}$ as defined in $(2.8), b\left(w_{1}, w_{2} ; \kappa\right):=$ $b^{\mathrm{U}}\left(w_{1}^{\kappa}, w_{2}^{\kappa}\right) / b^{\mathrm{U}}(1,1)$ can be a tail order function. Since $b^{\mathrm{U}}(1, \infty) \leq 1$, if $b^{\mathrm{U}}(1,1) \neq 0$, that is, the tail dependence parameter $\lambda_{U}$ in the sense of Section 2.1.10 of Joe (1997) satisfies $0<\lambda_{U} \leq 1$, then the tail order function $b\left(w_{1}, w_{2} ; \kappa\right)$ also satisfies $b(1, \infty ; \kappa)<\infty$; that is, $b(1, w ; \kappa)$ is bounded.

\subsection{Multivariate cases}

For multivariate cases with dimension $d \geq 3$, Proposition 2(2) of Hua and Joe (2011) implies that $\kappa_{I} \leq \kappa_{J}$ if $I \subseteq J \subseteq I_{d}$. It follows from the proof of Proposition 3.1 that, when we analyze HRV on $E^{(2)}$, we can determine the tail order $\kappa$ and the slowly varying function $\ell$ used in (3.6) based on all the bivariate margins. The smallest $\kappa$ among the tail orders for all the bivariate margins and its associated slowly varying functions would be used for (3.6); if more than one bivariate margin has smallest $\kappa$, then the tail order $\kappa$ and the largest slowly varying function for these bivariate margins would be used for (3.6). In this case, the upper exponent function on $E^{(2)}$ exists, and, by Proposition 3.1, all the upper tail order functions exist, but some of them could always be 0 .

If, for all multivariate margins $C_{I}$ with $I \subset I_{d}$ and $1<|I|$, the corresponding upper tail orders are the same and the associated slowly varying functions are tail equivalent up to some finite constants at $0^{+}$, then HRV on $E^{(2)}$ suffices; see Example 4.4 below for such a case with an Archimedean copula. Otherwise, we need to seek HRV on the other subcones $E^{(i)}, i=3, \ldots, d$; Example 4.5 below shows such a case with a Gaussian copula.

Example 4.4. (Archimedean copula based on the Laplace transform of the inverse gamma (also known as the ACIG copula; see Hua and Joe (2011)).) A positive continuous random vector $\boldsymbol{X}:=\left(X_{1}, \ldots, X_{d}\right)$ that has an ACIG copula with the dependence parameter $\beta>1$, and tail equivalent univariate margins $\bar{F}_{i} \in \mathrm{RV}_{-\alpha}, i \in I_{d}$, has HRV on $E^{(2)}$, because of Proposition 3.3 and the fact that all bivariate margins have upper tail order $\kappa=\min \{2, \beta\}$. The interesting thing is, if $1<\beta<2$ then, for any marginal copula $C_{I}$ with $I \subseteq I_{d}$, the upper tail order is $\kappa\left(C_{I}\right)=\beta$. Moreover, by reviewing the proof of Proposition 6 of Hua and Joe (2013), the associated slowly varying functions for upper tail order functions of the ACIG copula are constants. Therefore, by Proposition 3.1, all the upper tail order functions for $C_{I}$ exist and are not 0 , so there is no HRV on $E^{(i)}, i=3, \ldots, d$. 
Example 4.5. (Gaussian copula..) For the Gaussian copula

$$
C\left(u_{1}, \ldots, u_{d}\right):=\Phi_{\Sigma}\left(\Phi^{-1}\left(u_{1}\right), \ldots, \Phi^{-1}\left(u_{d}\right)\right),
$$

we assume that all the correlation coefficients are $\rho$. From Hua and Joe (2011), the tail order for such a copula is $\kappa=\mathbf{1}_{d} \Sigma^{-1} \mathbf{1}_{d}^{\top}=d /[1+(d-1) \rho]$, and all bivariate margins have upper tail order $\kappa_{\{i j\}}=2 /(1+\rho)$. If a positive, continuous random vector $\boldsymbol{X}$ has the Gaussian copula and the univariate margins $X_{i} \in \mathrm{RV}_{-\alpha}, i \in I_{d}$, then $X$ has HRV on $E^{(2)}$ by Proposition 3.3. The HRV structure here is different than that in Example 4.4. The Gaussian copula has different upper tail orders for margins with different dimensions, and there are still HRVs on $E^{(3)}, \ldots, E^{(d)}$. The upper exponent function on $E^{(2)}$ leads to the upper tail order functions always being 0 for $C_{I}$ with $3 \leq|I| \leq d$, and cannot provide useful information for the interior of $E^{(2)}$, so we need to seek HRV on the other subcones. Note that if some of the correlation coefficients are different then the smallest $\kappa_{\{i j\}}=2 /\left(1+\rho_{i j}\right)$ (corresponding to the largest $\rho_{i j}$ ) will be used in (3.6), and the upper exponent function becomes

$$
\begin{aligned}
& a^{\mathrm{U}}(\boldsymbol{w} ; 2, \kappa) \\
& \quad:=\lim _{u \rightarrow 0^{+}} \frac{\mathbb{P}\left(\bigcup_{\left\{i \neq j: \rho_{i j}=\max \left\{\rho_{k l}, k \neq l\right\}\right\}}\left\{U_{i}>1-u w_{i}, U_{j}>1-u w_{j}\right\}\right)}{u^{\kappa} \ell(u)}, \quad \boldsymbol{w} \in E_{0}^{(2)} .
\end{aligned}
$$

Remark 4.2. Note from the bivariate Gaussian copula with a negative $\rho$ that HRV on $E^{(2)}$ can even lead to $\kappa=\alpha_{2} / \alpha>2$. That is, HRV may lead to upper tail negative dependence. It is the ratio between the tail index for HRV and the tail index for MRV on $E^{(1)}$ that determines the value of the upper tail order, and, thus, the pattern of dependence in the upper tail.

\section{A mixture representation}

In this section we present a general mixture representation that can be used to generate tail order functions. One special case of the mixture representation corresponds to the Pickandstype representation (3.16). In comparison, the previous section just has tail order functions from some known copula families. If we were to use the tail order function for tail risk analysis or inferences on joint tail probabilities, it is important to have large classes of tail order functions as potential models.

Let $B \sim \operatorname{Bernoulli}(\pi), 0<\pi<1, Z \sim \operatorname{Pareto}\left(\alpha_{2}\right), \boldsymbol{R}:=\left(R_{1}, \ldots, R_{d}\right)$ be a random vector with CDF $F_{\boldsymbol{R}}$ with each margin being defined on [1, $)$, and let $X_{1}, \ldots, X_{d}$ be independent Pareto $\left(\alpha_{2}\right)$ random variables. Suppose that $B, Z, X_{1}, \ldots, X_{d}$, and $\left(R_{1}, \ldots, R_{d}\right)$ are all mutually independent. Moment assumptions on the $R_{j}$ are given below. Consider

$$
Y_{j}:=B R_{j} Z+(1-B) X_{j}^{\gamma}, \quad j=1, \ldots, d, 1<\gamma<d .
$$

We will show that the random vector $\boldsymbol{Y}:=\left(Y_{1}, \ldots, Y_{d}\right)$ has HRV on $E^{(d)}$ and the upper tail order is $\kappa=\gamma$. Moreover, for the bivariate case, if the probability measure generated by $F_{\boldsymbol{R}}$ only puts mass on $\delta \mathbb{N}_{2}$ then $F_{R_{1}}$ and $F_{R_{2}}$ correspond to the measures of $H_{1}$ and $H_{2}$ in Section 4 , respectively, up to some normalization constants.

For each univariate margin,

$$
\mathbb{P}\left(Y_{j}>y\right)=\pi \int_{1}^{\infty}\left(1+\frac{y}{r}\right)^{-\alpha_{2}} \mathrm{~d} F_{R_{j}}(r)+(1-\pi)\left(1+y^{1 / \gamma}\right)^{-\alpha_{2}} .
$$

Assume that $\int_{1}^{\infty} r^{\alpha_{2}} \mathrm{~d} F_{R_{j}}(r)<\infty$, that is, $\mathbb{E}\left[R_{j}^{\alpha_{2}}\right]$ is finite. Then $\bar{F}_{Y_{j}} \in \mathrm{RV}_{-\alpha_{2} / \gamma}$, and, as $u \rightarrow 0, \bar{F}_{Y_{j}}^{-1}(u) \sim[u /(1-\pi)]^{-\gamma / \alpha_{2}}$. Let $\alpha:=\alpha_{2} / \gamma$. Then, for each univariate margin $Y_{j}$, $\bar{F}_{Y_{j}} \in \mathrm{RV}_{-\alpha}$. 
For the joint survival probability,

$\mathbb{P}\left(Y_{j}>y_{j}, j=1, \ldots, d\right)=\pi \int_{1}^{\infty}\left[1+\max _{j}\left\{\frac{y_{j}}{r_{j}}\right\}\right]^{-\alpha_{2}} \mathrm{~d} F_{\boldsymbol{R}}(\boldsymbol{r})+(1-\pi) \prod_{j=1}^{d}\left(1+y_{j}^{1 / \gamma}\right)^{-\alpha_{2}}$.

The survival copula for $\boldsymbol{Y}$ is

$$
\begin{aligned}
C(\boldsymbol{u}) & =\mathbb{P}\left(Y_{j}>\bar{F}_{Y_{j}}^{-1}(u), j=1, \ldots, d\right) \\
& =\pi \int_{\mathbf{1}}^{\infty}\left[1+\max _{j}\left\{\frac{\bar{F}_{Y_{j}}^{-1}(u)}{r_{j}}\right\}\right]^{-\alpha_{2}} \mathrm{~d} F_{\boldsymbol{R}}(\boldsymbol{r})+(1-\pi) \prod_{j=1}^{d}\left(1+\left[\bar{F}_{Y_{j}}^{-1}(u)\right]^{1 / \gamma}\right)^{-\alpha_{2}} .
\end{aligned}
$$

Then

$$
\begin{aligned}
\lim _{u \rightarrow 0^{+}} & \frac{C(u \boldsymbol{w})}{u^{\gamma}} \\
& =\lim _{u \rightarrow 0^{+}} \frac{\pi \int_{\mathbf{1}}^{\infty}\left[1+\max _{j}\left\{\bar{F}_{Y_{j}}^{-1}\left(u w_{j}\right) / r_{j}\right\}\right]^{-\alpha_{2}} \mathrm{~d} F_{\boldsymbol{R}}(\boldsymbol{r})+(1-\pi) \prod_{j=1}^{d}\left(1+\left[\bar{F}_{Y_{j}}^{-1}\left(u w_{j}\right)\right]^{1 / \gamma}\right)^{-\alpha_{2}}}{u^{\gamma}} \\
& =\lim _{u \rightarrow 0^{+}} \frac{\pi \int_{\mathbf{1}}^{\infty}\left[1+\max _{j}\left\{\bar{F}_{Y_{j}}^{-1}\left(u w_{j}\right) / r_{j}\right\}\right]^{-\alpha_{2}} \mathrm{~d} F_{\boldsymbol{R}}(\boldsymbol{r})}{u^{\gamma}} \\
& =\pi(1-\pi)^{-\gamma} \int_{\mathbf{1}}^{\infty}\left[\min _{j}\left\{w_{j}^{\gamma / \alpha_{2}} r_{j}\right\}\right]^{\alpha_{2}} \mathrm{~d} F_{\boldsymbol{R}}(\boldsymbol{r}) \\
& =\pi(1-\pi)^{-\gamma} \int_{\mathbf{1}}^{\infty}\left[\min _{j}\left\{w_{j}^{1 / \alpha} r_{j}\right\}\right]^{\alpha_{2}} \mathrm{~d} F_{\boldsymbol{R}}(\boldsymbol{r}) .
\end{aligned}
$$

To get (5.2), a sufficient condition is that $\mathbb{E}\left[\left(\min _{j \in\{1, \ldots, d\}} R_{j}\right)^{\alpha_{2}}\right]<\infty$. Therefore, the tail order $\kappa$ satisfies $1<\kappa=\gamma$, and, by Proposition 3.3, $\boldsymbol{Y}$ has HRV on $E^{(2)}$.

Comparing (5.3) to (3.16) with $l=d$, we find that the $R_{i}$ here may play a similar role to the measure $S_{d}$. For the bivariate case, the upper tail order function coincides with the upper exponent function on $E^{(2)}$. Then, letting $\kappa=\gamma>1$,

$$
b\left(w_{1}, w_{2} ; \kappa\right)=\pi(1-\pi)^{-\kappa} \int_{1}^{\infty} \int_{1}^{\infty} \min \left\{w_{1}^{\kappa} r_{1}^{\alpha_{2}}, w_{2}^{\kappa} r_{2}^{\alpha_{2}}\right\} \mathrm{d} F_{\boldsymbol{R}}(\boldsymbol{r}) .
$$

Note that in the mixture representation (5.1), we do not specify the dependence structure between the $R_{j} \mathrm{~s}$. If in the bivariate case the probability measure generated by the distribution $F_{\boldsymbol{R}}$ of $R_{1}$ and $R_{2}$ only puts mass on the $L$-shape line $\delta \mathbb{N}_{2}$, then $F_{R_{1}}$ and $F_{R_{2}}$ correspond to the $H_{1}$ and $H_{2}$ measures in (4.4) and (4.3), respectively, up to some finite normalization constants. More generally, consider polar coordinates with angle $\theta \in[0, \pi / 2]$, radial variable $z$ with $r_{1}=z \cos \theta$ and $r_{2}=z \sin \theta$, and random variables $(Z, \Theta)$ obtained from $\left(R_{1}, R_{2}\right)$. The moment condition on $\mathbb{E}\left[\left(\min \left\{R_{1}, R_{2}\right\}\right)^{\alpha_{2}}\right]$ implies a moment condition on $Z$. Also, note that

$$
\begin{aligned}
w_{1}^{\kappa} r_{1}^{\alpha_{2}} \leq w_{2}^{\kappa} r_{2}^{\alpha_{2}} & \Longleftrightarrow \quad r_{1}^{\alpha_{2}} \leq\left(\frac{w_{2}}{w_{1}}\right)^{\kappa} r_{2}^{\alpha_{2}} \\
& \Longleftrightarrow \quad r_{2}^{\alpha_{2}} \geq r_{1}^{\alpha_{2}}\left(\frac{w_{1}}{w_{2}}\right)^{\kappa} \\
& \Longleftrightarrow \quad(\tan \theta)^{\alpha_{2}} \geq\left(\frac{w_{1}}{w_{2}}\right)^{\kappa} .
\end{aligned}
$$


Let $\vartheta\left(w_{1}, w_{2}\right):=\arctan \left[\left(w_{1} / w_{2}\right)^{\kappa / \alpha_{2}}\right], E_{2}(\theta):=\mathbb{E}\left(Z^{1+\alpha_{2}} \mid \Theta=\theta\right)$, and $F_{\Theta}$ be the CDF of the angle $\Theta$ obtained from $F_{\boldsymbol{R}}$. Then (5.4) implies that

$$
\begin{aligned}
\frac{b\left(w_{1}, w_{2} ; \kappa\right)}{\pi(1-\pi)^{-\kappa}=} & \int_{0}^{\vartheta\left(w_{1}, w_{2}\right)} \int_{z=1}^{\infty} w_{2}^{\kappa} z^{\alpha_{2}}(\sin \theta)^{\alpha_{2}} z \mathrm{~d} F_{Z, \Theta}(z, \theta) \\
& +\int_{\vartheta\left(w_{1}, w_{2}\right)}^{\pi / 2} \int_{z=1}^{\infty} w_{1}^{\kappa} z^{\alpha_{2}}(\cos \theta)^{\alpha_{2}} z \mathrm{~d} F_{Z, \Theta}(z, \theta) \\
= & w_{2}^{\kappa} \int_{0}^{\vartheta\left(w_{1}, w_{2}\right)} E_{2}(\theta)(\sin \theta)^{\alpha_{2}} \mathrm{~d} F_{\Theta}(\theta) \\
& +w_{1}^{\kappa} \int_{\vartheta\left(w_{1}, w_{2}\right)}^{\pi / 2} E_{2}(\theta)(\cos \theta)^{\alpha_{2}} \mathrm{~d} F_{\Theta}(\theta) .
\end{aligned}
$$

Hence, $b(\cdot)$ depends on $F_{\boldsymbol{R}}$ only through $E_{2}(\theta)$ and $F_{\Theta}$.

If $R_{1}$ and $R_{2}$ are comonotonic then (5.4) provides an upper bound for all bivariate upper tail order functions for random vectors that have the representation (5.1), that is, after appropriate normalization, $\min \left\{w_{1}^{\kappa}, w_{2}^{\kappa}\right\}$ can be an upper bound for all such bivariate upper tail order functions. Interesting tail order functions can result by taking specific parametric families for $R_{1}$ and $R_{2}$, for example, if $R_{1}$ and $R_{2}$ are independent Weibull random variables then the tail order function (hence, the associated intensity measure $v_{2}$ in Proposition 3.2) could incorporate negative as well as positive dependence, in the sense that $X_{1}$ and $X_{2}$, with the induced survival function

$$
\bar{F}_{X_{1}, X_{2}}\left(x_{1}, x_{2}\right):=\frac{b\left(x_{1}^{-1}, x_{2}^{-1}\right)}{b(1,1)}=\frac{\nu_{2}\left(\left(x_{1}, \infty\right) \times\left(x_{2}, \infty\right)\right)}{\nu_{2}((1, \infty) \times(1, \infty))},
$$

can be positively or negatively dependent.

For dimension $d \geq 3$, the upper tail order function coincides with the upper exponent function on $E^{(d)}$. Then (5.3) can still be used to explain the measure $S_{d}$ for HRV on $E^{(d)}$. That is, if the probability measure $F_{\boldsymbol{R}}$ only puts mass on $\delta \mathbb{N}_{d}$ then $F_{\boldsymbol{R}}$ corresponds to the measure $S_{d}$, up to some normalization constants. However, for HRV on $E^{(l)}$ with $2 \leq l<d$ and $d \geq 3$, the mixture representation (5.1) is not suitable to explain the measure $S_{d}$, and a related discussion for this case is already given in Section 4.2.

\section{Concluding remarks}

Depending on the tail index of univariate margins and the tail index for MRV on subcones of $E^{(i)}, i=2, \ldots, d$, the upper tail order $\kappa$ of the underlying copula can take any value greater than or equal to 1 . This means, in addition to the usual tail dependence, MRV may also incorporate the cases of intermediate tail dependence, tail orthant independence, and even tail negative dependence. If a $d$-dimensional random vector $\boldsymbol{X}$ has MRV on $E^{(1)}$ with copula $C$ and tail equivalent Pareto-like univariate margins with tail index $\alpha$, then the upper tail order for the marginal copula $C_{I}$ with $I \subseteq I_{d}$ and $2 \leq|I| \leq d$ is determined by the ratio of the tail index $\alpha_{|I|}$, if it exists, of MRV on $E^{(|I|)}$ and the tail index $\alpha$ for MRV on $E^{(1)}$.

Upper exponent functions on subcones for copulas have been introduced, and connections between those upper exponent functions and the intensity measures $v_{i}$ for HRV on the subcones have been established. The representations of Pickands type for upper exponent functions on subcones were obtained using an order-statistics-based product-measure decomposition. A mixture model for HRV on $E^{(d)}$ was constructed, for which, after appropriate normalization, the upper tail order function (i.e. the upper exponent function on $E^{(d)}$ ) can be represented by 
an integral with respect to a mixing probability measure that resembles the finite-directional measure in the Pickands representation.

We have built in this paper a bridge between the measure-theoretic MRV/HRV theory and the copula method for analyzing extremal dependence. On the one hand, the methods already developed in the MRV/HRV theory can be used to analyze tail dependence of copulas. On the other hand, there are many existing parametric copula families, and it is also relatively easier to create new parametric copula families. Therefore, the copula approach allows rich parametric distribution families to facilitate statistical analysis for multivariate heavy tail phenomena.

\section{Acknowledgement}

The authors want to thank the anonymous reviewer for the helpful comments and suggestions that improved the presentation of this paper.

\section{References}

Bingham, N. H., Goldie, C. M. And Teugels, J. L. (1987). Regular Variation (Encyclopedia Math. Appl. 27). Cambridge University Press.

De Haan, L. And Zhou, C. (2011). Extreme residual dependence for random vectors and processes. Adv. Appl. Prob. 43, 217-242.

Hashorva, E. (2010). On the residual dependence index of elliptical distributions. Statist. Prob. Lett. 80, 1070-1078.

Hua, L. AND JoE, H. (2011). Tail order and intermediate tail dependence of multivariate copulas. J. Multivariate Anal. 102, 1454-1471.

HuA, L. AND Joe, H. (2012a). Tail comonotonicity and conservative risk measures. ASTIN Bull.42, 601-629.

HuA, L. AND JoE, H. (2012b). Tail comonotonicity: properties, constructions, and asymptotic additivity of risk measures. Insurance Math. Econom. 51, 492-503.

Hua, L. And Joe, H. (2013). Intermediate tail dependence: a review and some new results. In Stochastic Orders in Reliability and Risk (Lecture Notes Statist. 208), Springer, New York, pp. 291-311.

JAWORSKI, P. (2004). On uniform tail expansions of bivariate copulas. Appl. Math. (Warsaw) 31, 397-415.

JAWORSKI, P. (2006). On uniform tail expansions of multivariate copulas and wide convergence of measures. Appl. Math. (Warsaw) 33, 159-184.

Joe, H. (1997). Multivariate Models and Dependence Concepts (Monogr. Statist. Appl. Prob. 73). Chapman \& Hall, London.

Joe, H., Li, H. And Nikoloulopoulos, A. K. (2010). Tail dependence functions and vine copulas. J. Multivariate Anal. 101, 252-270.

Klüppelberg, C., Kuhn, G. And Peng, L. (2008). Semi-parametric models for the multivariate tail dependence function - the asymptotically dependent case. Scand. J. Statist. 35, 701-718.

LEDFORD, A. W. AND TAWN, J. A. (1996). Statistics for near independence in multivariate extreme values. Biometrika 83, 169-187.

Li, H. AND Sun, Y. (2009). Tail dependence for heavy-tailed scale mixtures of multivariate distributions. J. Appl. Prob. 46, 925-937.

MaUliK, K. AND Resnick, S. (2004). Characterizations and examples of hidden regular variation. Extremes 7, 31-67.

Mitra, A. And Resnick, S. (2010). Hidden regular variation: Detection and estimation. Preprint. Available at http:/ arxiv.org/abs/1001.5058v2.

Mitra, A. AND ReSNick, S. I. (2011). Hidden regular variation and detection of hidden risks. Stoch. Models 27, $591-614$.

Nikoloulopoulos, A. K., JoE, H. AND Li, H. (2009). Extreme value properties of multivariate $t$ copulas. Extremes 12, 129-148.

Resnick, S. I. (1987). Extreme Values, Regular Variation, and Point Processes (Appl. Prob. 4). Springer, New York.

ReSNick, S. I. (2007). Heavy-tail Phenomena. Springer, New York.

SkLAR, A. (1959). Fonctions de répartition à $n$ dimensions et leurs marges. Publ. Inst. Statist. Univ. Paris 8, $229-231$. 